MATHEMATICS OF COMPUTATION

Volume 69, Number 231, Pages 1071-1098

S 0025-5718(99)01114-X

Article electronically published on March 10, 1999

\title{
MULTIHOMOGENEOUS NEWTON METHODS
}

\author{
JEAN-PIERRE DEDIEU AND MIKE SHUB
}

\begin{abstract}
We study multihomogeneous analytic functions and a multihomogeneous Newton's method for finding their zeros. We give a convergence result for this iteration and we study two examples: the evaluation map and the generalized eigenvalue problem.
\end{abstract}

\section{INTRODUCTION AND MAIN RESULTS}

1.1. Introduction. In a series of papers, Shub [8] and Shub and Smale [9], [10], [11], [12, [13, studied a projective version of Newton's method for homogeneous systems. Their particular focus was the problem of finding zeros of systems of $n$ homogeneous polynomial equations in $n+1$ unknowns. In this paper we study multihomogeneous functions and a multihomogeneous Newton's method for finding their zeros.

Here are three examples of multihomogeneous functions. Let $\mathcal{H}_{d}$ be the space of homogeneous polynomials of degree $d$ defined on $\mathbb{C}^{n}$. Let $(d)=\left(d_{1}, \ldots, d_{m}\right)$ and $\mathcal{H}_{(d)}=\prod_{i=1}^{m} \mathcal{H}_{d_{i}}$. So elements of $\mathcal{H}_{(d)}$ represent polynomial functions $f: \mathbb{C}^{n} \rightarrow \mathbb{C}^{m}$, where $f=\left(f_{1}, \ldots, f_{m}\right)$ and $f_{i}$ is homogeneous of degree $d_{i}$. The evaluation map

$$
e v: \mathcal{H}_{(d)} \times \mathbb{C}^{n} \rightarrow \mathbb{C}^{m}
$$

$e v(f, x)=f(x)$, is multihomogeneous. Each coordinate function of $e v$ is linear in $f$ and homogeneous of degree $d_{i}$ in $x$.

A second example is given by the generalized eigenvalue problem. Let $A, B$ : $\mathbb{C}^{n} \rightarrow \mathbb{C}^{n}$ be linear operators. Then

$$
F_{(A, B)}: \mathbb{C}^{2} \times \mathbb{C}^{n} \rightarrow \mathbb{C}^{n}, \quad F_{(A, B)}(\alpha, \beta, x)=(\alpha B-\beta A)(x),
$$

is bilinear, i.e. it is linear in $(\alpha, \beta)$ and linear in $x$. The generalized eigenvalue problem is to find the zeros of $F_{(A, B)}$. then

A third example is given by homogenization. If $f: \mathbb{E} \rightarrow \mathbb{F}$ is complex analytic

$$
\hat{f}: \mathbb{E} \times \mathbb{C}^{\star} \rightarrow \mathbb{F}, \quad \hat{f}(x, t)=f(x / t),
$$

is complex analytic and homogeneous of degree 0 .

In general let $\mathbb{E}_{1}, \ldots, \mathbb{E}_{k}$ be complex or real vector spaces and $\mathbb{F}=\mathbb{C}^{m}$ or $\mathbb{R}^{m}$. Let $\mathbb{E}=\mathbb{E}_{1} \times \ldots \times \mathbb{E}_{k}$ and $((d))=\left(\left(d_{1}\right), \ldots,\left(d_{k}\right)\right),\left(d_{i}\right)=\left(d_{1 i}, \ldots, d_{k i}\right)$ for

Received by the editor October 16, 1997 and, in revised form, July 22, 1998.

1991 Mathematics Subject Classification. Primary 65H10, 15 A99.

This paper was completed when the first author was visiting at the IBM T.J. Watson Research Center in August 1997.

The second author was partially supported by an NSF grant. 
$i=1, \ldots, m$. Then $f: \mathbb{E} \rightarrow \mathbb{F}$ is multihomogeneous of degree $((d))$ if and only if the $i$-th coordinate function satisfies

$$
f_{i}\left(\lambda_{1} x_{1}, \ldots, \lambda_{k} x_{k}\right)=\prod_{j=1}^{k} \lambda_{j}^{d_{j i}} f_{i}\left(x_{1}, \ldots, x_{k}\right)
$$

for $\left(x_{1}, \ldots, x_{k}\right) \in \mathbb{E}$ and $\left(\lambda_{1}, \ldots, \lambda_{k}\right)$ a $k$-tuple of scalars, i.e., $\left(\lambda_{1}, \ldots, \lambda_{k}\right) \in \mathbb{G}=$ $\mathbb{C}^{k}$ or $\mathbb{R}^{k}$ as the case may be.

We assume throughout that $f$ is analytic. The domain of $f$ may be an open subset of $\mathbb{E}$, but with abuse of notation we continue to write $f: \mathbb{E} \rightarrow \mathbb{F}$.

The multihomogeneous projective Newton iteration we define below is defined on $\mathbb{E}$ but is invariant under the natural identifications which define the product of the projective spaces $\mathbb{P}\left(\mathbb{E}_{1}\right) \times \ldots \times \mathbb{P}\left(\mathbb{E}_{k}\right)$. Indeed this is much of our motivation in defining Newton's iteration as we do, but it is important to keep in mind that implementations of the method reside in $\mathbb{E}$ itself !

For the rest of this paper we will assume that $\mathbb{E}, \mathbb{F}$ and $\mathbb{G}$ are complex and finite dimensional vector spaces and that $\mathbb{E}_{i}$ has an Hermitian product $\langle,\rangle_{i}$. For the case where $\mathbb{E}, \mathbb{F}$ and $\mathbb{G}$ are real we would replace the Hermitian product by an inner product. Also, we denote

$$
\mathbb{E}^{\star}=\left(\mathbb{E}_{1} \backslash\{0\}\right) \times \ldots \times\left(\mathbb{E}_{k} \backslash\{0\}\right) .
$$

If $\lambda=\left(\lambda_{1}, \ldots, \lambda_{k}\right) \in \mathbb{G}$, we define

$$
\times \lambda: \mathbb{E} \rightarrow \mathbb{E}
$$

by

$$
\times \lambda x=\left(\lambda_{1} x_{1}, \ldots, \lambda_{k} x_{k}\right) .
$$

Then $\mathbb{P}\left(\mathbb{E}_{1}\right) \times \ldots \times \mathbb{P}\left(\mathbb{E}_{k}\right)$ is the quotient of $\mathbb{E}^{\star}$ by the action of $\mathbb{G}^{\star}=(\mathbb{C} \backslash\{0\}) \times$ $\ldots \times(\mathbb{C} \backslash\{0\})$ ( $k$ times). For $x \in \mathbb{E}^{\star}, x=\left(x_{1}, \ldots, x_{k}\right)$, we let $x_{i}^{\perp}$ be the Hermitian complement of $x_{i}$ in $\mathbb{E}_{i}$,

$$
x^{\perp}=\prod_{i=1}^{k} x_{i}^{\perp} \subset \mathbb{E} \quad \text { and } \quad V_{x}=\left(x^{\perp}\right)^{\perp} \subset \mathbb{E} .
$$

Notice that $V_{x}$ is also the subspace of $\mathbb{E}$ spanned by the vectors $\left(0, \ldots, x_{i}, \ldots, 0\right)$, $i=1, \ldots, k$. The dimension of $V_{x}$ is $k$ since $x \in \mathbb{E}^{\star}$. For each $i, x_{i}^{\perp}$ is a natural representative of the tangent space $T_{x_{i}} \mathbb{P}\left(\mathbb{E}_{i}\right)$, and hence $x^{\perp}$ is a natural representative of the tangent space

$$
T_{x}\left(\prod_{i=1}^{k} \mathbb{P}\left(\mathbb{E}_{i}\right)\right)=\prod_{i=1}^{k} T_{x_{i}}\left(\mathbb{P}\left(\mathbb{E}_{i}\right)\right) .
$$

If $x=\times \lambda y$ for $\lambda \in \mathbb{G}^{\star}$ and $v \in y^{\perp}$, then $\times \lambda v \in x^{\perp}$ represents the same tangent vector in $T_{x}\left(\prod \mathbb{P}\left(\mathbb{E}_{i}\right)\right)$.

We now define an Hermitian structure on $\mathbb{E}$ depending on $x$ and hence on $x^{\perp}$ by

$$
\langle v, w\rangle_{x}=\sum_{i=1}^{k} \frac{\left\langle v_{i}, w_{i}\right\rangle_{i}}{\left\langle x_{i}, x_{i}\right\rangle_{i}}
$$

for $x \in \mathbb{E}^{\star}, v$ and $w \in \mathbb{E}$. If $\lambda \in \mathbb{G}^{\star}$, then $\times \lambda$ maps $x^{\perp}$ onto $(\times \lambda x)^{\perp}$ and

$$
\langle\times \lambda v, \times \lambda w\rangle_{(\times \lambda x)}=\langle v, w\rangle_{x},
$$


so $\langle,\rangle_{x}$ defines an Hermitian product on $T_{x}\left(\mathbb{P}\left(\mathbb{E}_{1}\right) \times \ldots \times \mathbb{P}\left(\mathbb{E}_{k}\right)\right)$. Condition $(*)$ says that $\times \lambda$ is an isometry from $x^{\perp}$ to $(\times \lambda x)^{\perp}$ as well as $\mathbb{E}$ to $\mathbb{E}$ with their given Hermitian products.

We are now ready to define the multihomogeneous projective Newton iteration for $f$. We denote this map as $N_{f}: \prod_{i} \mathbb{P}\left(\mathbb{E}_{i}\right) \hookleftarrow$.

\section{Definition 1.}

$$
N_{f}(x)=f(x)-\left(\left.D f(x)\right|_{x^{\perp}}\right)^{\dagger} f(x) .
$$

Here $\left(\left.D f(x)\right|_{x^{\perp}}\right)^{\dagger}$ is the Moore-Penrose inverse of the restriction of $D f(x)$ to $x^{\perp}$. We recall that if $A: V_{1} \rightarrow V_{2}$ is a linear map between two finite dimensional complex vector spaces with Hermitian products, then the Moore-Penrose inverse of $A$ maps $V_{2}$ to $V_{1}$ and is the composition of two maps

$$
A^{\dagger}: V_{2} \rightarrow V_{1}, \quad A^{\dagger}=i \Pi
$$

where $\Pi$ is the Hermitian projection of $V_{2}$ onto $i m A$ and $i: i m A \rightarrow V_{1}$ is the right inverse of $A$ whose image in $V_{1}$ is the Hermitian complement of $\operatorname{ker} A$. If $A$ is surjective then $A^{\dagger}=A^{\star}\left(A A^{\star}\right)^{-1}$, where $A^{\star}$ is the adjoint of $A$. In this paper we only take Moore-Penrose inverses of surjective linear maps, unless otherwise noted.

$N_{f}$ is of course naturally defined on $\mathbb{E}$; we use $N_{f}$ to denote this map as well. From the context it should be clear which map we mean and whether we mean Newton's iteration, projective Newton's iteration or multihomogeneous projective Newton's iteration.

Proposition 1. $N_{f}$ is well-defined, i.e., if $y=\times \lambda x$ for $x \in \mathbb{E}^{\star}$ and $\lambda \in \mathbb{G}^{\star}$, then $N_{f}(y)=\times \lambda N_{f}(x)$.

For the proof we use a lemma which will be useful later. Let $\Lambda=\left(\Lambda_{1}, \ldots, \Lambda_{k}\right)$, where $\Lambda_{i}=\prod_{j=1}^{k} \lambda_{j}^{d_{j i}}$ and $f$ has degree $((d))$. Then

Lemma 1. 1. $f(\times \lambda x)=\times \Lambda f(x)$.

2. $D f(\times \lambda x) \times \lambda=\times \Lambda D f(x)$.

3. $D^{l} f(\times \lambda x)(\times \lambda, \ldots, \times \lambda)=\times \Lambda D^{l} f(x)$.

4. $(\times \lambda)^{-1}\left(\left.D f(\times \lambda x)\right|_{(\times \lambda x)^{\perp}}\right)^{\dagger}=\left(\left.D f(x)\right|_{x^{\perp}}\right)^{\dagger}(\times \Lambda)^{-1}$.

Proof of Lemma 1. 1 is the definition of multihomogeneity. 2 and 3 then follow from the chain rule. 4 follows from 2 since $(\times \lambda)$ is an isometry which maps ker $D f(x)$ to ker $D f(\times \lambda x)$ and hence $i m\left(\left.D f(x)\right|_{x^{\perp}}\right)^{\dagger}$ to $\operatorname{im}\left(\left.D f(\times \lambda x)\right|_{(\times \lambda x)^{\perp}}\right)^{\dagger}$.

Proof of Proposition 1. We have

$$
\begin{aligned}
\left(\left.D f(\times \lambda x)\right|_{(\times \lambda x)^{\perp}}\right)^{\dagger} f(\times \lambda x) & =(\times \lambda)\left(\left.D f(x)\right|_{x^{\perp}}\right)^{\dagger}(\times \Lambda)^{-1}(\times \Lambda) f(x) \\
& =(\times \lambda)\left(\left.D f(x)\right|_{x^{\perp}}\right)^{\dagger} f(x)
\end{aligned}
$$

by 4 and 1 of Lemma 1 .

Our analysis of the multihomogeneous Newton method closely follows Smale [14]. There are three important quantities associated to $f$ and $x$, which we now define.

Definition 2. 1. $\gamma(f, x)=\max \left(1, \sup _{k \geq 2}\left\|\left(\left.D f(x)\right|_{x^{\perp}}\right)^{\dagger} D^{k} f(x) / k !\right\|_{x}^{1 /(k-1)}\right)$.

2. $\beta(f, x)=\left\|\left(\left.D f(x)\right|_{x^{\perp}}\right)^{\dagger} f(x)\right\|_{x}$.

3. $\alpha(f, x)=\beta(f, x) \gamma(f, x)$. 
In the definition of $\gamma(f, x),\|\|_{x}$ is the operator norm with respect to $\langle,\rangle_{x}$.

We now verify that $\alpha(f, x), \beta(f, x)$ and $\gamma(f, x)$ are defined on $\mathbb{P}\left(\mathbb{E}_{1}\right) \times \ldots \times \mathbb{P}\left(\mathbb{E}_{k}\right)$.

Proposition 2. For any $x \in \mathbb{E}^{\star}$ and $\lambda \in \mathbb{G}^{\star}$ we have $\star(f, x)=\star(f, \times \lambda x)$ with $\star \in\{\alpha, \beta, \gamma\}$.

Proof of Proposition 2. By Lemma 1

$$
(\times \lambda)\left(\left.D f(x)\right|_{x^{\perp}}\right)^{\dagger} f(x)=\left(\left.D f(\times \lambda x)\right|_{(\times \lambda x)^{\perp}}\right)^{\dagger} f(\times \lambda x)
$$

as in Proposition 1, and

$$
\left(\left.D f(x)\right|_{x^{\perp}}\right)^{\dagger} D^{k} f(x)=(\times \lambda)^{-1}\left(\left.D f(\times \lambda x)\right|_{(\times \lambda x)^{\perp}}\right)^{\dagger} D^{k} f(\times \lambda x)(\times \lambda, \ldots, \times \lambda) .
$$

Since $\times \lambda$ is an isometry, we obtain the required result.

We recall that for $i=1, \ldots, k$ the Riemannian distance in $\mathbb{P}\left(\mathbb{E}_{i}\right)$ is given by

$$
d_{R}\left(x_{i}, y_{i}\right)=\arccos \frac{\left|\left\langle x_{i}, y_{i}\right\rangle_{i}\right|}{\left\|x_{i}\right\|_{i}\left\|y_{i}\right\|_{i}},
$$

and in $\mathbb{P}\left(\mathbb{E}_{1}\right) \times \ldots \times \mathbb{P}\left(\mathbb{E}_{k}\right)$ by

$$
d_{R}(x, y)=\left(\sum_{i=1}^{k} d_{R}\left(x_{i}, y_{i}\right)^{2}\right)^{1 / 2}
$$

where $x=\left(x_{1}, \ldots, x_{k}\right)$ and $y=\left(y_{1}, \ldots, y_{k}\right) \in \mathbb{E}^{\star}$. Here and throughout we identify $x_{i} \in \mathbb{E}_{i} \backslash\{0\}$ and $x \in \mathbb{E}^{\star}$ with their equivalence classes in $\mathbb{P}\left(\mathbb{E}_{i}\right)$ and $\mathbb{P}\left(\mathbb{E}_{1}\right) \times \ldots \times$ $\mathbb{P}\left(\mathbb{E}_{k}\right)$ respectively.

Our main theorems concerning the convergence of the multihomogeneous Newton iteration are summarized in the following subsections and proved in $\S 2$.

\section{2. $\alpha$-theorem.}

Theorem 1. There is a universal constant $\alpha_{u}>0$ with the following property: for any multihomogeneous system $f: \mathbb{E} \rightarrow \mathbb{F}$ and $x \in \mathbb{E}^{\star}$, if $\alpha(f, x) \leq \alpha_{u}$ and $\left.D f(x)\right|_{x^{\perp}}$ (the restriction of $D f(x)$ to $x^{\perp}$ ) is onto, then the multihomogeneous Newton sequence

$$
x_{0}=x, \quad x_{k+1}=x_{k}-\left(\left.D f\left(x_{k}\right)\right|_{x_{k}^{\perp}}\right)^{\dagger} f\left(x_{k}\right)
$$

satisfies

$$
\left\|x_{k+1}-x_{k}\right\|_{x_{k}} \leq\left(\frac{1}{2}\right)^{2^{k}-1} \beta(f, x)
$$

for any $k \geq 0$. This sequence converges to a zero $\zeta \in \mathbb{E}^{\star}$ of $f$, and

$$
d_{R}\left(\zeta, x_{k}\right) \leq \sigma\left(\frac{1}{2}\right)^{2^{k}-1} \beta(f, x)
$$

with

$$
\sigma=\sum_{i=0}^{\infty}\left(\frac{1}{2}\right)^{2^{i}-1}=1.6328 \ldots
$$

We can take $\alpha_{u}=1 / 137$. 
$\alpha$-theorems are available in several different contexts. This approach of Newton's methods finds its origins in a paper by S. Smale [12] for analytic functions $f: \mathbb{E} \rightarrow \mathbb{F}$ with $\mathbb{E}$ and $\mathbb{F}$ Banach spaces. Sharpened results are given by Royden 7], ShubSmale [9] and Wang [16].

Newton's method can be generalized to search for zeros of maps $f: \mathbb{R}^{n} \rightarrow$ $\mathbb{R}^{m}, n \geq m$, using the Moore-Penrose inverse of the derivative: $N_{f}^{M P}(x)=x-$ $D f(x)^{\dagger} f(x)$. This method appears in the book of Allgower and Georg [1. An $\alpha$-theorem is given in this context by Shub and Smale in 12 .

Projective Newton's method has been proposed by Shub in [8] for homogeneous systems $f: \mathbb{C}^{n+1} \rightarrow \mathbb{C}^{n}$ and is defined by $N_{f}^{P}(x)=x-\left.D f(x)\right|_{x^{\perp}} ^{-1} f(x)$. An $\alpha-$ theorem has been given by Malajovich in [6]. In the same paper this author also studies Moore-Penrose projective Newton's iteration $N_{f}^{M P P}(x)=x-D f(x)^{\dagger} f(x)$ for such homogeneous systems.

\section{3. $\gamma$-theorem.}

Theorem 2. There are universal constants $\gamma_{u}$ and $c_{u}>0$ with the following properties: Let $\zeta \in \mathbb{E}^{\star}$ be a zero of $f$ with $D f(\zeta)$ onto and $x \in \mathbb{E}^{\star}$. If

$$
\|x-\zeta\|_{\zeta} \gamma(f, \zeta) \leq \gamma_{u}
$$

then the multihomogeneous Newton sequence converges to a zero $\zeta^{\prime} \in \mathbb{E}^{\star}$ of $f$, and

$$
d_{R}\left(\zeta^{\prime}, x_{k}\right) \leq \sigma\left(\frac{1}{2}\right)^{2^{k}-1} \beta(f, x)
$$

Moreover

$$
d_{R}\left(\zeta^{\prime}, x\right) \leq 3\|x-\zeta\|_{\zeta}
$$

and

$$
d_{R}\left(\zeta^{\prime}, N_{f}(x)\right) \leq c_{u} \gamma(f, \zeta)\|x-\zeta\|_{\zeta}^{2}
$$

We have not tried to find the largest possible values for $\alpha_{u}$ or $\gamma_{u}$. The proof of Theorem 2 crudely shows that we can take $\gamma_{u}=.00005$.

Corollary 1. There is a universal constant $\delta_{u}$ with the following property: Let $\zeta \in \mathbb{E}^{\star}$ be a zero of $f$ with $\operatorname{Df}(\zeta)$ onto and $x \in \mathbb{E}^{\star}$. If

$$
d_{R}(x, \zeta) \gamma(f, \zeta) \leq \delta_{u}
$$

then the multihomogeneous Newton sequence converges to a zero $\zeta^{\prime} \in \mathbb{E}^{\star}$ of $f$, and

$$
d_{R}\left(\zeta^{\prime}, x_{k+1}\right) \leq\left(\frac{1}{2}\right)^{2^{k}} d_{R}(\zeta, x)
$$

This theorem gives the size of the attraction basin around a given zero of the system $f$. The affine case is treated by Shub-Smale in [9] and in [12] for overdetermined systems and Moore-Penrose Newton's iteration. For homogeneous systems $f: \mathbb{C}^{n+1} \rightarrow \mathbb{C}^{n}$ see Blum-Cucker-Shub-Smale [2], Chapter 14, Theorem 1. The $\gamma$-theorem is the main ingredient to prove complexity results for path-following methods. It will be used in the other sections. 
1.4. Newton's method for the evaluation map. Let $\mathcal{H}_{d}$ be the space of homogeneous polynomials of degree $d$ defined on $\mathbb{C}^{n}, n>1$. Let $(d)=\left(d_{1}, \ldots, d_{m}\right)$ and $\mathcal{H}_{(d)}=\prod_{i=1}^{m} \mathcal{H}_{d_{i}}$. The evaluation map

$$
\text { ev }: \mathcal{H}_{(d)} \times \mathbb{C}^{n} \rightarrow \mathbb{C}^{m}
$$

$e v(f, x)=f(x)$, is bihomogeneous: each coordinate function $e v\left(f_{i}, x\right)$ is linear in $f_{i}$ and homogeneous of degree $d_{i}$ in $x$.

The Hermitian structure over $\mathcal{H}_{(d)}$ is the product structure: for $f=\left(f_{1}, \ldots, f_{m}\right)$ and $g=\left(g_{1}, \ldots, g_{m}\right)$ we define

$$
\langle f, g\rangle=\sum_{i=1}^{m}\left\langle f_{i}, g_{i}\right\rangle
$$

and

$$
\left\langle f_{i}, g_{i}\right\rangle=\sum_{|\alpha|=d_{i}}\left(\begin{array}{c}
d_{i} \\
\alpha
\end{array}\right)^{-1} a_{i, \alpha} \bar{b}_{i, \alpha}
$$

with $f_{i}(z)=\sum_{|\alpha|=d_{i}} a_{i, \alpha} z^{\alpha}, g_{i}(z)=\sum_{|\alpha|=d_{i}} b_{i, \alpha} z^{\alpha}, \alpha=\left(\alpha_{1}, \ldots, \alpha_{n}\right),|\alpha|=\alpha_{1}+$ $\ldots+\alpha_{n}$ and $\left(\begin{array}{c}d_{i} \\ \alpha\end{array}\right)=\frac{d_{i} !}{\alpha_{1} ! \ldots \alpha_{n} !}$.

Let us denote

$$
V=\left\{(g, y) \in \mathcal{H}_{(d)} \times \mathbb{C}^{n}: \operatorname{ev}(g, y)=0\right\} .
$$

For any $(f, x) \in \mathcal{H}_{(d)}$ close enough to $V$, multihomogeneous Newton's method constructs a sequence $N_{e v}^{(k)}(f, x)$ which converges quadratically to a unique element in $V$ denoted by $M_{e v}(f, x)$. This defines a function which projects a neighborhood of $V$ onto $V$ itself. By Theorem 2, the size of this neighborhood is controlled by

$$
\gamma(e v, V)=\max _{(g, y) \in V} \gamma(e v, g, y)
$$

We have obtained the following estimate

Theorem 3. $\gamma(e v, V) \leq \frac{D(D-1)}{2}(1+\sqrt{m})$ with $D=\max d_{i}$.

The properties of $M_{e v}(f, x)$ are summarized in the following theorem.

Theorem 4. Let $(f, x) \in \mathcal{H}_{(d)} \times \mathbb{C}^{n}$ be such that

$$
d_{R}((f, x) ; V) \leq \frac{2 \delta_{u}}{D(D-1)(1+\sqrt{m})} .
$$

Let $(g, y) \in V$ satisfy $d_{R}((f, x) ; V)=d_{R}((f, x) ;(g, y))$. Then the multihomogeneous Newton's sequence $N_{e v}^{(k)}(f, x)$ converges to $M_{e v}(f, x) \in V$. Moreover

$$
d_{R}\left(M_{e v}(f, x) ;(f, x)\right) \leq 3\left(\frac{\|f-g\|^{2}}{\|g\|^{2}}+\frac{\|x-y\|^{2}}{\|x\|^{2}}\right)^{1 / 2}
$$

and

$$
d_{R}\left(M_{e v}(f, x) ; N_{e v}^{(k)}(f, x)\right) \leq\left(\frac{1}{2}\right)^{2^{k}}\left(\frac{\|f-g\|^{2}}{\|g\|^{2}}+\frac{\|x-y\|^{2}}{\|x\|^{2}}\right)^{1 / 2}
$$


1.5. Path-following. In the following theorem we analyse the complexity of a path-following method to solve a system of equations approximately. The context we deal with is the following: for any $t \in[0,1]$ let $f_{t}: \mathbb{E} \rightarrow \mathbb{F}$ be a multihomogeneous system depending smoothly on $t$. We also suppose that $\operatorname{dim} \mathbb{F}=\operatorname{dim} x^{\perp}$ for $x \in \mathbb{E}^{\star}$; that is, after disregarding the homogenizing directions, the number of equations and the number of unknowns are the same. Let $\zeta_{t}$ be a smooth curve in $\mathbb{E}^{\star}$ such that $f_{t}\left(\zeta_{t}\right)=0$ and $\left.D f_{t}\left(\zeta_{t}\right)\right|_{\zeta_{t}^{\perp}}$ is an isomorphism. We associate to a subdivision $0=t_{0}<t_{1}<\ldots<t_{p}=1$ a sequence $x_{i}$ defined by

$$
x_{0}=\zeta_{0} \quad \text { and } \quad x_{i+1}=N_{f_{t_{i+1}}}\left(x_{i}\right) .
$$

When the subdivision size $\max \left|t_{i+1}-t_{i}\right|$ is small enough, then

$$
d_{R}\left(x_{i}, \zeta_{t_{i}}\right) \gamma\left(f_{t_{i}}, \zeta_{t_{i}}\right) \leq \delta_{u}
$$

so that, by Theorem $2, x_{i}$ may be taken as the starting point for a multihomogeneous Newton sequence $N_{f_{t_{i}}}^{k}\left(x_{i}\right)$ converging quadratically towards $\zeta_{t_{i}}$.

The complexity of this path-following method is given by $p$, the number of points in the subdivision. Before we state our result we have to introduce more invariants:

\section{Definition 3.}

$$
\begin{gathered}
\gamma=\max _{0 \leq t \leq 1} \gamma\left(f_{t}, x_{t}\right), \\
\mu=\max _{0 \leq t \leq 1}\left\|D f_{t}\left(\zeta_{t}\right)^{\dagger}\right\|_{\zeta_{t}},
\end{gathered}
$$

and $L$ is the length of the curve $t \in[0,1] \rightarrow f_{t}$.

$\mu$ is the condition number of the curve $t \in[0,1] \rightarrow\left(f_{t}, \zeta_{t}\right)$. Our main result asserts that the complexity of this path-following method depends mainly on the product $\mu \gamma L$.

Theorem 5. There is a partition $0=t_{0}<t_{1}<\ldots<t_{p}=1$ with

$$
p=\left\lceil\frac{2}{\delta_{u}} \gamma \mu L\right\rceil
$$

such that, for each $i=0 \ldots p$ the sequence defined by

$$
x_{0}=\zeta_{0} \text { and } x_{i+1}=N_{f_{t_{i+1}}}\left(x_{i}\right)
$$

satisfies

$$
d_{R}\left(x_{i}, \zeta_{t_{i}}\right) \gamma\left(f_{t_{i}}, \zeta_{t_{i}}\right) \leq \delta_{u}
$$

Remark. Theorem 5 states the existence of a partition without giving a hint as to how to construct one. For practical implementations a good strategy may consist in taking $t_{i+1}=t_{i}+\lambda\left(t_{i}-t_{i-1}\right)$. In the first step take $\lambda=2$, i.e., double the step length. If the corresponding iterate $x_{i+1}$ is not an approximate zero for $f_{i+1}$, change $\lambda$ in $\lambda / 2$ and compute a new $x_{i+1}$.

There is a considerable literature concerning path-following methods. The book of Allgower and Georg [1] is a good introduction to this subject. We follow here the lines of Shub and Smale: [9] for the affine case, 12 for the affine underdetermined case. The case of sparse polynomial systems is studied by Dedieu in [4]. 
1.6. Newton's method for the generalized eigenvalue problem. Let $(A, B)$ $\in \mathcal{M}_{n}(\mathbb{C}) \times \mathcal{M}_{n}(\mathbb{C})$ be a matrix pair. A pair is called singular when the homogeneous polynomial $P_{(A, B)}(\alpha, \beta)=\operatorname{det}(\beta A-\alpha B)$ is identically 0 . Otherwise it is said to be regular. In such a case this polynomial has degree $n$ and its zeros consist in $n$ lines through the origin. These lines are the eigenvalues of the pair $(A, B)$, and the nontrivial solutions $x \in \mathbb{C}^{n}$ of the equation

$$
(\beta A-\alpha B) x=0
$$

are the corresponding eigenvectors.

In order to compute approximately the eigenvalues and eigenvectors of this matrix pair we introduce

$$
F_{(A, B)}: \mathbb{C}^{2} \times \mathbb{C}^{n} \rightarrow \mathbb{C}^{n}, \quad F_{(A, B)}(\alpha, \beta, x)=(\beta A-\alpha B) x,
$$

which is a bihomogeneous polynomial with degree 1 in each variable. Multihomogeneous Newton's iterate is thus equal to

$$
N_{F_{(A, B)}}(\alpha, \beta, x)=(\alpha, \beta, x)-\left.D F_{(A, B)}(\alpha, \beta, x)\right|_{(\alpha, \beta, x)^{\perp}} ^{\dagger}(\beta A-\alpha B) x .
$$

A more precise description of this iterate is given in Section 2.6.

Our objective is here to describe the complexity of a path-following method to compute approximately an eigenpair (i.e. an eigenvalue, eigenvector pair) associated with a matrix pair. Let $\left(A_{0}, B_{0}\right)$ and $\left(A_{1}, B_{1}\right)$ be two regular matrix pairs. We consider two smooth curves

$$
t \in[0,1] \rightarrow(1-t)\left(A_{0}, B_{0}\right)+t\left(A_{1}, B_{1}\right)=\left(A_{t}, B_{t}\right)
$$

and

$$
t \in[0,1] \rightarrow\left(\alpha_{t}, \beta_{t}, x_{t}\right) \in \mathbb{C}^{2} \times \mathbb{C}^{n}
$$

so that

$$
\left(\beta_{t} A_{t}-\alpha_{t} B_{t}\right) x_{t}=0 .
$$

We also suppose that $\left(\alpha_{t}, \beta_{t}\right)$ is a simple eigenvalue for the pair $\left(A_{t}, B_{t}\right)$. The pathfollowing method consists in the following:

$$
0=t_{0}<t_{1}<\ldots<t_{p}=1
$$

is a given subdivision and

$$
\begin{gathered}
\left(a_{0}, b_{0}, z_{0}\right)=\left(\alpha_{0}, \beta_{0}, x_{0}\right), \\
\left(a_{i+1}, b_{i+1}, z_{i+1}\right)=N_{i+1}\left(a_{i}, b_{i}, z_{i}\right), \quad i=0, \ldots, p-1 .
\end{gathered}
$$

Here $N_{i}$ is the multihomogeneous Newton's iterate associated with the matrix pair $\left(A_{t_{i}}, B_{t_{i}}\right)$. Starting from the eigenpair $\left(\alpha_{0}, \beta_{0}, x_{0}\right)$ of $\left(A_{0}, B_{0}\right)$, we obtain an approximate eigenpair $\left(a_{p}, b_{p}, z_{p}\right)$ for $\left(A_{1}, B_{1}\right)$. Here, approximate means

$$
\alpha\left(F_{\left(A_{1}, B_{1}\right)},\left(a_{p}, b_{p}, z_{p}\right)\right) \leq \alpha_{u},
$$

so that, by Theorem 1 , the sequence $\left.N_{p}^{k}\left(a_{p}, b_{p}, z_{p}\right)\right), k \geq 1$, converges quadratically to $\left(\alpha_{1}, \beta_{1}, x_{1}\right)$.

Our main theorem in this section gives a bound for a sufficient $p$ in terms of the condition number of the path. This last quantity is defined by 


\section{Definition 4.}

$$
\mu=\max _{0 \leq t \leq 1} \mu\left(A_{t}, B_{t}, \alpha_{t}, \beta_{t}, x_{t}\right)
$$

with

$$
\mu(A, B, \alpha, \beta, x)=\left\|\left.D F_{(A, B)}(\alpha, \beta, x)\right|_{(\alpha, \beta, x)^{\perp}} ^{\dagger}\right\|_{(\alpha, \beta, x)} .
$$

$\mu(A, B, \alpha, \beta, x)$ is the condition number for the generalized eigenvalue problem and $\mu$ the condition number for the path.

Theorem 6. There is a partition $0=t_{0}<t_{1}<\ldots<t_{p}=1$ with

$$
\begin{gathered}
p=\left\lceil\frac{1}{\delta_{u}} \max (r, s)\right\rceil, \\
r=2 \mu\left(\left\|A_{0}-A_{1}\right\|_{F}^{2}+\left\|B_{0}-B_{1}\right\|_{F}^{2}\right)^{1 / 2}, \\
s=\mu^{2} \max \left(\left(\left\|A_{0}\right\|^{2}+\left\|B_{0}\right\|^{2}\right)^{1 / 2},\left(\left\|A_{1}\right\|^{2}+\mid B_{1} \|^{2}\right)^{1 / 2}\right) \\
\times\left(\left\|A_{0}-A_{1}\right\|_{F}^{2}+\left\|B_{0}-B_{1}\right\|_{F}^{2}\right)^{1 / 2},
\end{gathered}
$$

such that $\left(a_{p}, b_{p}, z_{p}\right)$ is an approximate eigenpair for $\left(A_{1}, B_{1}\right)$.

Here $\|A\|$ is the spectral norm and $\|A\|_{F}$ the Frobenius norm.

Remark. Such a path-following method might be combined with a "divide and conquer" strategy as in Li [5]:

$$
A_{0}=\left(\begin{array}{cc}
A_{11} & 0 \\
0 & A_{22}
\end{array}\right), \quad A_{1}=\left(\begin{array}{cc}
A_{11} & A_{12} \\
A_{21} & A_{22}
\end{array}\right),
$$

and similarly for $B_{0}$ and $B_{1}$. See, also, Li's discussion of the number of solutions of $(\beta A-\alpha B) x=0$ considered as a quadratic or a bihomogeneous system of equations. The bihomogeneous context seems more natural.

The remainder of this paper is organized as follow: in Section 2.1 we give some results about the angle between two subspaces in a Euclidean or Hermitian space. These results will be useful later. We present them in a separate section to make reading easier. In Section 2.i, $2 \leq i \leq 6$, we give the proofs of the theorems presented in Section 1.i.

\section{Proofs of TheOrems}

2.1. Angles between subspaces in a Hermitian space. We denote by $\mathbb{E}$ a complex Hermitian space or a real Euclidean space. To measure the distance between two vector subspaces $V$ and $W$ in $\mathbb{E}$ it is useful to consider the following quantity:

\section{Definition 5.}

$$
d(V, W)=\max _{v \in V^{\star}} \min _{w \in W} \frac{\|v-w\|}{\|v\|} .
$$

This number is the maximum of the sine of a given vector $v \in V$ with its orthogonal projection on $W$. It also has the following characterizations $\left(\Pi_{X}\right.$ denotes the orthogonal projection on $X$ ): 
Proposition 3. 1. $d(V, W)=\left\|\Pi_{W^{\perp}} \Pi_{V}\right\|$.

2. $d(V, W)=d\left(W^{\perp}, V^{\perp}\right)$.

3. $d(V, W)=d\left(V \cap(V \cap W)^{\perp}, W \cap(V \cap W)^{\perp}\right)$.

Proof. 1 goes as follows:

$d(V, W)=\max _{\substack{v \in V \\\|v\|=1}}\left\|\left(i d-\Pi_{W}\right) v\right\|=\max _{\substack{v \in V \\\|v\| \leq 1}}\left\|\Pi_{W^{\perp}} v\right\|=\max _{\|v\|=1}\left\|\Pi_{W^{\perp}} \Pi_{V} v\right\|=\left\|\Pi_{W^{\perp}} \Pi_{V}\right\|$.

2 is a consequence of 1 since the norms of an operator and its transpose are equal.

Let us prove the third assertion. For any $v \in V$ we write it as

$$
v=v_{1}+v_{2} \in(V \cap W) \oplus\left(V \cap(V \cap W)^{\perp}\right) .
$$

Then

$$
\Pi_{W} v=w_{1}+w_{2} \in(V \cap W) \oplus\left(W \cap(V \cap W)^{\perp}\right)
$$

with $w_{1}=v_{1}$ and $w_{2}=\Pi_{W \cap(V \cap W)^{\perp}}\left(v_{2}\right)$.

The proof of Proposition 3.1 may be found in Stewart-Sun [15] with other useful properties of $d(V, W)$. This number measures the distance of $V$ from $W$, but is not stricto sensu a distance because in general $d(V, W)$ and $d(W, V)$ are not equal. For this reason it is convenient to define

$$
\delta(V, W)=\max (d(V, W), d(W, V)) .
$$

$\delta$ is a (true) distance in the set of vector subspaces in $\mathbb{E}$. We also have

Proposition 4. 1. $0 \leq d(V, W) \leq 1$.

2. $d(V, W)=0$ if and only if $V \subset W$.

3. $d(V, W)<1$ if and only if $V \cap W^{\perp}=\{0\}$.

4. $d\left(V_{1}, V_{3}\right) \leq d\left(V_{1}, V_{2}\right)+d\left(V_{2}, V_{3}\right)$.

5. If $V_{1} \subset V_{2}$, then $d\left(V_{1}, W\right) \leq d\left(V_{2}, W\right)$, and if $W_{1} \subset W_{2}$, then $d\left(V, W_{2}\right) \leq$ $d\left(V, W_{1}\right)$.

6. $d\left(V, W_{1}+W_{2}\right) \leq \min \left(d\left(V, W_{1}\right), d\left(V, W_{2}\right)\right)$.

7. If $V_{1}$ and $V_{2}$ are orthogonal, then $d\left(V_{1} \oplus V_{2}, W\right) \leq d\left(V_{1}, W\right)+d\left(V_{2}, W\right)$ and $d\left(V_{1} \oplus V_{2}, W\right) \leq \sqrt{2} \max \left(d\left(V_{1}, W\right), d\left(V_{2}, W\right)\right)$.

8. If $\operatorname{dim} V=\operatorname{dim} W$, then $d(V, W)=d(W, V)$.

These properties (more precisely, 2, 4 and 8) show that $d(V, W)$ defines a distance (sticto sensu) on the Grassmannian manifold $\mathbb{G}_{n, p}$ of $p$-dimensional vector subspaces in $\mathbb{C}^{n}$. When $\operatorname{dim} V \neq \operatorname{dim} W$ then, by $3, \delta(V, W)=1$, while, when $\operatorname{dim} V=\operatorname{dim} W, d(V, W)=d(W, V)=\delta(V, W)$. In the sequel we only use $d(V, W)$.

Proof. 1 to 7.1 are staightforward. We now prove 7.2. If $v_{1}$ and $v_{2}$ are orthogonal then $\left\|v_{1}\right\|+\left\|v_{2}\right\| \leq \sqrt{2}\left\|v_{1}+v_{2}\right\|$. So, if $V_{1}$ and $V_{2}$ are orthogonal,

$$
\begin{aligned}
d\left(V_{1} \oplus V_{2}, W\right) & =\left\|\Pi_{W^{\perp}}\left(v_{1}+v_{2}\right)\right\| \leq\left\|\Pi_{W^{\perp}} v_{1}\right\|+\left\|\Pi_{W^{\perp}} v_{2}\right\| \\
& \leq d\left(V_{1}, W\right)\left\|v_{1}\right\|+d\left(V_{2}, W\right)\left\|v_{2}\right\| \\
& \leq \max \left(d\left(V_{1}, W\right), d\left(V_{2}, W\right)\right)\left(\left\|v_{1}\right\|+\left\|v_{2}\right\|\right) \\
& \leq \sqrt{2} \max \left(d\left(V_{1}, W\right), d\left(V_{2}, W\right)\right)\left\|v_{1}+v_{2}\right\| .
\end{aligned}
$$

To prove 8 we first remark that $d(V, W)$ is the largest singular value of $\Pi_{W} \perp \Pi_{V}=$ $\left(i d-\Pi_{W}\right) \Pi_{V}=\Pi_{V}-\Pi_{W} \Pi_{V}$, and similarly $d(W, V)$ is the largest singular value of $\Pi_{W}-\Pi_{V} \Pi_{W}$. Let us introduce a unitary transformation $Q$ such that $Q^{2}=i d$ 
and $Q V=W$. The existence of such an involution will be proved at the end of this section. We have $\Pi_{W}=Q \Pi_{V} Q$, so that

$$
\Pi_{W} \Pi_{V}=\Pi_{V}-\Pi_{W} \Pi_{V}=\Pi_{V}-Q \Pi_{V} Q \Pi_{V}
$$

and similarly

$$
\Pi_{V \perp} \Pi_{W}=Q\left(\Pi_{V}-Q \Pi_{V} Q \Pi_{V}\right) Q .
$$

Thus $\Pi_{W^{\perp}} \Pi_{V}$ and $\Pi_{V}{ } \Pi_{W}$ have the same singular values and $d(V, W)=d(W, V)$.

Appendix to Section 2.1. Let $V$ and $W$ be two vector subspaces in $\mathbb{E}$ with the same dimension $n$. The proof of Proposition 4.8 requires the existence of an involution $Q$ in $\mathbb{E}$ which sends $V$ onto $W$. The existence of such an involution may be well known, but we have not found it in the literature. A proof of the fact may be derived from the CS decomposition for partitionned unitary matrices, see Stewart-Sun [15]. We give here a concise and elegant construction due to A. J. Hoffman.

We only consider the case $\mathbb{E}=\mathbb{C}^{2 n}, V \cap W=\{0\}$ and $V \oplus W=\mathbb{C}^{2 n}$. The general case is easily deduced from this one. We also suppose that $V$ is spanned by the first $n$ vectors of the canonical basis in $\mathbb{C}^{2 n}$. Let us introduce two $2 n \times n$ matrices:

$$
S=\left(\begin{array}{c}
I_{n} \\
0
\end{array}\right) \text { and } T=\left(\begin{array}{l}
A \\
C
\end{array}\right)
$$

such that the columns of $T$ span $W$ and $T$ is orthonormal. Notice that $S$ spans $V$. Let us write $A U=H$, the polar decomposition of $A$ : $U$ is unitary and $H$ positive semidefinite; $T U=\left(\begin{array}{c}H \\ B^{\star}\end{array}\right)$ also spans $W$. We remark now that $B^{\star}$ is nonsingular: if $B^{\star} x=0$ then $T U x=\left(\begin{array}{c}H x \\ 0\end{array}\right)$, so that $T U x \in V \cap W=\{0\}$. This gives $x=0$, since $U$ is unitary and $T$ orthonormal. $B$ is also nonsingular. Let us now consider the following $2 n \times 2 n$ matrix:

$$
Q=\left(\begin{array}{cc}
H & B \\
B^{\star} & -B^{-1} H B
\end{array}\right)
$$

We have

$$
H^{2}+B B^{\star}=\left(\begin{array}{ll}
H & B
\end{array}\right)\left(\begin{array}{c}
H \\
B^{\star}
\end{array}\right)=U^{\star} T^{\star} T U=I_{n},
$$

so that

$$
H B B^{\star}=H\left(I_{n}-H^{2}\right)=\left(I_{n}-H^{2}\right) H=B B^{\star} H .
$$

This yields $B^{-1} H B=B^{\star} H B^{-\star}$, and consequently $Q$ is Hermitian. Using the same argument, we see easily that $Q^{2}=I_{2 n}$, so that $Q$ is an involution. To complete the proof we remark that $Q S=\left(\begin{array}{c}H \\ B^{\star}\end{array}\right)=T U$ spans $W$. 
2.2. $\alpha$-theorem. In this section we give a proof of Theorem 1 . It is split into fourteen different lemmas. We first recall some notations and introduce some new ones. We let $x_{i}^{\perp}$ be the Hermitian complement of $x_{i}$ in $\mathbb{E}_{i}$,

$$
x^{\perp}=\prod_{i=1}^{k} x_{i}^{\perp} \subset \mathbb{E} \text { and } V_{x}=\left(x^{\perp}\right)^{\perp} \subset \mathbb{E} \text {. }
$$

We also introduce

$$
W_{x}=i m\left(\left.D f(x)\right|_{x^{\perp}}\right)^{\dagger} \text { and } W_{x}^{\perp}=V_{x} \oplus\left(\operatorname{ker} D f(x) \cap x^{\perp}\right),
$$

where the $\oplus$ is orthogonal. We also use frequently for $x, \zeta \in \mathbb{E}^{\star}$ and $y \in \mathbb{E}$

$$
u_{x}=\|y-x\|_{x} \gamma(f, x), \quad u_{\zeta}=\|y-\zeta\|_{\zeta} \gamma(f, \zeta)
$$

and the function

$$
\psi(u)=2 u^{2}-4 u+1, \quad 0 \leq u \leq 1-\frac{\sqrt{2}}{2} .
$$

This function is decreasing from 1 at $u=0$ to 0 at $u=1-\sqrt{2} / 2$. We first start with a linear algebra lemma.

Lemma 2.a. Let $X$ and $Y$ be Hermitian spaces and $A, B: X \rightarrow Y$ linear operators with $B$ onto. If

$$
\left\|B^{\dagger}(B-A)\right\| \leq \lambda<1
$$

then $A$ is onto and

$$
\left\|A^{\dagger} B\right\|<\frac{1}{1-\lambda}
$$

Proof. Let us denote $C=B-A$. We have $\left\|B^{\dagger} C\right\| \leq \lambda<1$, so that $i d_{X}-B^{\dagger} C$ is nonsingular and

$$
\left\|\left(i d_{X}-B^{\dagger} C\right)^{-1}\right\|<\frac{1}{1-\lambda}
$$

by a classical argument. Because $B$ is onto we have $B B^{\dagger}=i d_{Y}$, so that

$$
(B-C)^{\dagger} B\left(i d_{X}-B^{\dagger} C\right)=(B-C)^{\dagger}(B-C)=\Pi
$$

with $\Pi$ the orthogonal projection on $(\operatorname{ker} A)^{\perp}$. Thus

$$
\left\|A^{\dagger} B\right\|=\left\|(B-C)^{\dagger} B\right\|=\left\|\Pi\left(i d_{X}-B^{\dagger} C\right)^{-1}\right\| \leq\|\Pi\|\left\|\left(i d_{X}-B^{\dagger} C\right)^{-1}\right\|<\frac{1}{1-\lambda} .
$$

Moreover,

$$
A=B-C=B-B B^{\dagger} C=B\left(i d_{X}-B^{\dagger} C\right)
$$

is the composition of $B$ onto, and $\left(i d_{X}-B^{\dagger} C\right)$ nonsingular. Thus $A$ is onto and we are done.

Lemma 2.b. Let $x \in \mathbb{E}^{\star}$ and $y \in \mathbb{E}$ be given such that $\left.D f(x)\right|_{x^{\perp}}$ is onto and $u_{x}<1-\frac{\sqrt{2}}{2}$. Then $\left.D f(y)\right|_{x^{\perp}}$ is onto and

$$
\left\|\left.\left(\left.D f(y)\right|_{x^{\perp}}\right)^{\dagger} D f(x)\right|_{x^{\perp}}\right\|_{x} \leq \frac{\left(1-u_{x}\right)^{2}}{\psi\left(u_{x}\right)} .
$$


Proof. We have

$$
D f(y)=D f(x)+\sum_{k \geq 2} k \frac{D^{k} f(x)}{k !}(y-x)^{k-1},
$$

so that

$$
\left(\left.D f(x)\right|_{x^{\perp}}\right)^{\dagger}\left(\left.D f(y)\right|_{x^{\perp}}-\left.D f(x)\right|_{x^{\perp}}\right)=\left.\sum_{k \geq 2} k\left(\left.D f(x)\right|_{x^{\perp}}\right)^{\dagger} \frac{D^{k} f(x)}{k !}(y-x)^{k-1}\right|_{x^{\perp}} .
$$

If we take the operator norm of both sides, we get

$$
\begin{aligned}
\left\|\left(\left.D f(x)\right|_{x^{\perp}}\right)^{\dagger}\left(\left.D f(y)\right|_{x^{\perp}}-\left.D f(x)\right|_{x^{\perp}}\right)\right\|_{x} & \leq \sum_{k \geq 2} k \gamma(f, x)^{k-1}\|y-x\|_{x}^{k-1} \\
& =\sum_{k \geq 2} k u_{x}^{k-1}=\frac{1}{\left(1-u_{x}\right)^{2}}-1,
\end{aligned}
$$

and this number is $<1$ since $u_{x}<1-\frac{\sqrt{2}}{2}$. By Lemma 2.a $\left.D f(y)\right|_{x^{\perp}}$ is onto, and

$$
\left\|\left.\left(\left.D f(y)\right|_{x^{\perp}}\right)^{\dagger} D f(x)\right|_{x^{\perp}}\right\|_{x} \leq \frac{1}{1-\left(\frac{1}{\left(1-u_{x}\right)^{2}}-1\right)}=\frac{\left(1-u_{x}\right)^{2}}{\psi\left(u_{x}\right)} .
$$

Lemma 3.a. We have $f(\zeta)=0$ if and only if $V_{\zeta} \subseteq \operatorname{ker} D f(\zeta)$. In this case

$$
W_{\zeta}^{\perp}=\operatorname{ker} D f(\zeta)=V_{\zeta} \oplus\left(\operatorname{ker} D f(\zeta) \cap \zeta^{\perp}\right)
$$

and

$$
W_{\zeta}=i m\left(\left.D f(\zeta)\right|_{\zeta^{\perp}}\right)^{\dagger}=\operatorname{imDf}(\zeta)^{\dagger} .
$$

Proof. Since $f_{i}: \mathbb{E} \rightarrow \mathbb{C}$ is multihomogeneous with degrees $d_{1 i}, \ldots, d_{k i}$, then (Euler formula)

$$
D f_{i}(x)(\times \lambda x)=f_{i}(x) \sum_{j=1}^{k} d_{j i} \lambda_{j}
$$

and this proves the first assertion. Since $W_{\zeta}=i m\left(\left.D f(\zeta)\right|_{\zeta^{\perp}}\right)^{\dagger}$ is the orthogonal complement in $\zeta^{\perp}$ of $\left.\operatorname{ker} D f(\zeta)\right|_{\zeta^{\perp}}$, we have

$$
W_{\zeta}=\zeta^{\perp} \cap\left(\zeta^{\perp} \cap \operatorname{ker} D f(\zeta)\right)^{\perp}=\zeta^{\perp} \cap\left(V_{\zeta} \oplus \operatorname{ker} D f(\zeta)^{\perp}\right),
$$

so that

$$
W_{\zeta}^{\perp}=V_{\zeta} \oplus\left(\zeta^{\perp} \cap \operatorname{ker} D f(\zeta)\right) .
$$

Since $V_{\zeta} \subset \operatorname{ker} D f(\zeta)$, this gives

$$
W_{\zeta}^{\perp}=\operatorname{ker} D f(\zeta) \quad \text { and } \quad W_{\zeta}=\operatorname{ker} D f(\zeta)^{\perp}=i m D f(\zeta)^{\dagger} .
$$

Lemma 3.b. If $f(\zeta)=0$ and $u_{\zeta}<1-\frac{\sqrt{2}}{2}$, then

$$
d_{\zeta}\left(\operatorname{ker} D f(y) \cap y^{\perp}, \operatorname{ker} D f(\zeta) \cap \zeta^{\perp}\right) \leq \frac{1}{\left(1-u_{\zeta}\right)^{2}}-1+u_{\zeta} .
$$


Proof. We have $\zeta^{\perp}=\left(\operatorname{ker} D f(\zeta) \cap \zeta^{\perp}\right) \oplus W_{\zeta}$. Given $v \in \operatorname{ker} D f(y) \cap y^{\perp}$, we get

$$
0=D f(y) v=D f(\zeta) v+\sum_{k \geq 2} k \frac{D^{k} f(\zeta)}{k !}(y-\zeta)^{k-1} v .
$$

Now let $v=v_{1}+v_{2}$ with $v_{1} \in W_{\zeta}^{\perp}$ and $v_{2} \in W_{\zeta}$. Then $D f(\zeta)^{\perp} D f(\zeta) v=v_{2}$ and

$$
-v_{2}=\sum_{k \geq 2} k D f(\zeta)^{\dagger} \frac{D^{k} f(\zeta)}{k !}(y-\zeta)^{k-1} v
$$

so that

$$
\left\|v-v_{1}\right\|_{\zeta}=\left\|v_{2}\right\|_{\zeta} \leq\left(\frac{1}{\left(1-u_{\zeta}\right)^{2}}-1\right)\|v\|_{\zeta}
$$

as in the proof of Lemma 2.b. By Lemma 3.a, the component of $v_{1}$ in $V_{\zeta}$ is also the component of $v$ in $V_{\zeta}$, and its norm is bounded by

$$
d_{\zeta}\left(y^{\perp}, \zeta^{\perp}\right)\|v\|_{\zeta}=d_{\zeta}\left(V_{\zeta}, V_{y}\right)\|v\|_{\zeta} \leq u_{\zeta}\|v\|_{\zeta} ;
$$

the last inequality is a consequence of Lemma 4.a, and the equality follows from Proposition 3.2.

Lemma 3.c. If $f(\zeta)=0, \frac{1}{\left(1-u_{\zeta}\right)^{2}}-1+u_{\zeta} \leq 1$ and $D f(\zeta)$ is onto, then $\left.D f(y)\right|_{y^{\perp}}$ is onto.

Proof. By Lemma 3.b,

$$
d_{\zeta}\left(\operatorname{ker} D f(y) \cap y^{\perp}, \operatorname{ker} D f(\zeta) \cap \zeta^{\perp}\right)<1 .
$$

Thus, by Proposition 4.3

$$
\left(\operatorname{ker} D f(y) \cap y^{\perp}\right) \cap\left(\operatorname{ker} D f(\zeta) \cap \zeta^{\perp}\right)^{\perp}=\{0\},
$$

so that

$$
\operatorname{dim} \operatorname{ker} D f(y) \cap y^{\perp} \leq \operatorname{dim} \operatorname{ker} D f(\zeta) \cap \zeta^{\perp} \leq \operatorname{dim} \operatorname{ker} D f(\zeta)
$$

and we are done.

Lemma 4.a. $d_{x}\left(V_{x}, V_{y}\right) \leq\|x-y\|_{x} \leq u_{x}$.

Proof. Let $v \in V_{x}$ be given with $v=\times \lambda x$. With $w=\times \lambda y$ we have

$$
\begin{aligned}
\|v-w\|_{x} & =\left(\sum_{i} \frac{\left\|\lambda_{i}\left(x_{i}-y_{i}\right)\right\|_{i}^{2}}{\left\|x_{i}\right\|_{i}^{2}}\right)^{1 / 2} \\
& \leq \sum_{i} \frac{\left\|\lambda_{i}\left(x_{i}-y_{i}\right)\right\|_{i}}{\left\|x_{i}\right\|_{i}} \leq\left(\sum_{i}\left|\lambda_{i}\right|^{2}\right)^{1 / 2}\|x-y\|_{x}=\|v\|_{x}\|x-y\|_{x} .
\end{aligned}
$$

This gives $d_{x}\left(V_{x}, V_{y}\right) \leq\|x-y\|_{x}$, and this last quantity is $\leq u_{x}$ since $\gamma(f, x) \geq 1$.

Lemma 4.b. If $f(\zeta)=0$ and $u_{\zeta}<1-\frac{\sqrt{2}}{2}$, then

$$
d_{\zeta}\left(\operatorname{ker} D f(y), W_{\zeta}^{\perp}\right) \leq u_{\zeta} \phi\left(u_{\zeta}\right), \text { with } \phi(u)=\frac{2-u}{2 \psi(u)} .
$$


Proof. By Lemma 3.a, $\operatorname{ker} D f(\zeta) \oplus W_{\zeta}$ is under the hypothesis an orthogonal direct sum decomposition of $\mathbb{E}$. Let $v \in \operatorname{ker} D f(y)$ and $v=v_{1}+v_{2}$, where $v_{1} \in \operatorname{ker} D f(\zeta)$ and $v_{2} \in W_{\zeta} . D f(y) v=0$ implies that $D f(y) v_{1}=-D f(y) v_{2}$ and that $D f(\zeta)^{\dagger} D f(y) v_{1}=-D f(\zeta)^{\dagger} D f(y) v_{2}$. Now we estimate the norms of both sides of the equation:

$$
\begin{aligned}
\left\|D f(\zeta)^{\dagger} D f(y) v_{1}\right\|_{\zeta} & =\left\|D f(\zeta)^{\dagger}\left(D f(\zeta)+\sum_{k \geq 2} k \frac{D^{k} f(\zeta)}{k !}(y-\zeta)^{k-1}\right) v_{1}\right\|_{\zeta} \\
& \leq\left(\frac{1}{\left(1-u_{\zeta}\right)^{2}}-1\right)\left\|v_{1}\right\|_{\zeta}, \\
\left\|D f(\zeta)^{\dagger} D f(y) v_{2}\right\|_{\zeta} & =\left\|D f(\zeta)^{\dagger}\left(D f(\zeta)+\sum_{k \geq 2} k \frac{D^{k} f(\zeta)}{k !}(y-\zeta)^{k-1}\right) v_{2}\right\|_{\zeta} \\
& \geq\left\|v_{2}\right\|_{\zeta}-\left(\frac{1}{\left(1-u_{\zeta}\right)^{2}}-1\right)\left\|v_{2}\right\|_{\zeta} .
\end{aligned}
$$

Let $u \phi_{1}(u)=\frac{1}{(1-u)^{2}}-1$; then it follows that

$$
\left\|v_{1}\right\|_{\zeta} \geq \frac{1-u_{\zeta} \phi_{1}\left(u_{\zeta}\right)}{u_{\zeta} \phi_{1}\left(u_{\zeta}\right)}\left\|v_{2}\right\|_{\zeta}
$$

and that

$$
d_{\zeta}\left(\operatorname{ker} D f(y), W_{\zeta}^{\perp}\right) \leq \frac{u_{\zeta} \phi_{1}\left(u_{\zeta}\right)}{1-u_{\zeta} \phi_{1}\left(u_{\zeta}\right)}=u_{\zeta} \phi\left(u_{\zeta}\right)
$$

It remains to estimate $d_{\zeta}\left(W_{\zeta}^{\perp}, W_{y}^{\perp}\right)$, which we do in the next lemma with $\phi$ as above.

Lemma 4.c. If $f(\zeta)=0$ then

$$
d_{\zeta}\left(W_{\zeta}^{\perp}, W_{y}^{\perp}\right) \leq \sqrt{2} u_{\zeta} \phi\left(u_{\zeta}\right)
$$

Proof. Indeed,

$$
d_{\zeta}\left(W_{\zeta}^{\perp}, W_{y}^{\perp}\right)=d_{\zeta}\left(V_{y} \oplus\left(\operatorname{ker} D f(y) \cap y^{\perp}\right), W_{\zeta}^{\perp}\right),
$$

which by Proposition 4.7

$$
\leq \sqrt{2} \max \left(d_{\zeta}\left(V_{y}, W_{\zeta}^{\perp}\right), d_{\zeta}\left(\operatorname{ker} D f(y) \cap y^{\perp}, W_{\zeta}^{\perp}\right)\right)
$$

and by Lemma 4.b

$$
\leq \sqrt{2} \max \left(u_{\zeta}, u_{\zeta} \phi\left(u_{\zeta}\right)\right)=\sqrt{2} u_{\zeta} \phi\left(u_{\zeta}\right)
$$

Lemma 5. If $d_{\zeta}\left(\operatorname{ker} D f(\zeta), W_{y}^{\perp}\right) \leq \frac{\sqrt{3}}{2}$ and $\left.D f(y)\right|_{y^{\perp}}$ is onto, then

$$
\left\|\left(\left.D f(y)\right|_{y^{\perp}}\right)^{\dagger} D f(y)\right\|_{\zeta} \leq 2 .
$$


Proof. If $w_{1} \in \operatorname{ker} D f(y)^{\perp}$ then

$$
\left(\left.D f(y)\right|_{y^{\perp}}\right)^{\dagger} D f(y) w_{1}=w_{2},
$$

where $w_{2} \in W_{y}$ and there is a $v \in \operatorname{ker} D f(y)$ such that $w_{1}+v=w_{2}$. Then $\Pi_{\text {ker } D f(y)} w_{2}=v$, and by Proposition 3 and the hypotheses $\|v\|_{\zeta} \leq \frac{\sqrt{3}}{2}\left\|w_{2}\right\|_{\zeta}$, so

$$
\left\|w_{2}\right\|_{\zeta}^{2}=\left\|w_{1}\right\|_{\zeta}^{2}+\|v\|_{\zeta}^{2} \leq\left\|w_{1}\right\|_{\zeta}^{2}+\frac{3}{4}\left\|w_{2}\right\|_{\zeta}^{2}
$$

and $\left\|w_{2}\right\|_{\zeta} \leq 2\left\|w_{1}\right\|_{\zeta}$, which was to be proved.

Lemma 6. For any $x, y \in \mathbb{E}^{\star}$ let us define $c(x, y)=\max _{i}\left(\left\|x_{i}\right\|_{i} /\left\|y_{i}\right\|_{i}\right)$ and $c(y, x)=\max _{i}\left(\left\|y_{i}\right\|_{i} /\left\|x_{i}\right\|_{i}\right)$. Then

1. $\|v\|_{y} \leq c(x, y)\|v\|_{x}$ for any $v \in \mathbb{E}$.

2. $\|B\|_{y} \leq c(x, y) c(y, x)^{k}\|B\|_{x}$ for any $k$-linear operator $B: \mathbb{E}^{k} \rightarrow \mathbb{E}$.

Moreover when $u_{x}<1$ we have

3. $c(x, y) \leq \frac{1}{1-u_{x}}$ and $c(y, x) \leq 1+u_{x}$.

Proof. Assertions 1 and 2 are easy. To prove 3, notice that

$$
c(x, y)=\max _{i} \frac{\left\|x_{i}\right\|_{i}}{\left\|y_{i}\right\|_{i}} \leq \max _{i} \frac{1}{1-\frac{\left\|x_{i}-y_{i}\right\|_{i}}{\left\|x_{i}\right\|_{i}}} \leq \frac{1}{1-u_{x}}
$$

since

$$
\frac{\left\|x_{i}-y_{i}\right\|_{i}}{\left\|x_{i}\right\|_{i}} \leq\|y-x\|_{x} \leq u_{x}<1
$$

Moreover,

$$
c(y, x)=\max _{i} \frac{\left\|y_{i}\right\|_{i}}{\left\|x_{i}\right\|_{i}} \leq\|y\|_{x} \leq 1+\|y-x\|_{x} \leq 1+u_{x}
$$

Let $x \in \mathbb{E}^{\star}$ be given such that $\left.D f(x)\right|_{x^{\perp}}$ is onto. We use affine $\alpha$-theory (see Shub-Smale [12, Theorem 1.4) to conclude, if $\alpha(f, x) \leq \alpha_{0}$, the existence of a zero $\zeta$ of $f$ such that the Newton iterates

$$
x_{0}=x, \quad x_{k+1}=x_{k}-\left(\left.D f\left(x_{k}\right)\right|_{x^{\perp}}\right)^{\dagger} f\left(x_{k}\right)
$$

are such that $\left.D f\left(x_{k}\right)\right|_{x^{\perp}}$ is onto, converge to a zero $\zeta$ of $f$, and for all $k \geq 1$

$$
\left\|x_{k+1}-x_{k}\right\|_{x} \leq\left(\frac{1}{2}\right)^{2^{k}-1}\left\|x_{1}-x_{0}\right\|_{x} .
$$

With

$$
\sigma=\sum_{i=0}^{\infty}\left(\frac{1}{2}\right)^{2^{i}-1}=1.63284 \ldots
$$

Here $\alpha_{0}$ is a universal constant. According to Shub-Smale [9] and [12] we can take $\alpha_{0}=(13-3 \sqrt{17}) / 4=0.15767 \ldots$ We have the following:

Lemma 7. Let $x \in \mathbb{E}^{\star}$ be given such that $\left.D f(x)\right|_{x^{\perp}}$ is onto and $\alpha(f, x) \leq \alpha_{0}$. Let us denote $y=x_{1}=x-\left(\left.D f(x)\right|_{x^{\perp}}\right)^{\dagger} f(x)$. Then

$$
\begin{gathered}
\|\zeta-x\|_{x} \leq \sigma\|y-x\|_{x}=\sigma \beta(f, x), \\
\|\zeta-y\|_{x} \leq(\sigma-1)\|y-x\|_{x}=(\sigma-1) \beta(f, x) .
\end{gathered}
$$


The proof is easy, and is left to the reader.

Lemma 8. With the same hypotheses as in Lemma 7, there is a universal constant $\tau>0$, approximately equal to $23.78463 \ldots$, such that $u_{\zeta} \leq \tau u_{x}$.

Proof. Since $f(\zeta)=0$ we have $V_{\zeta} \subset \operatorname{ker} D f(\zeta)$, so that $\left(\left.D f(\zeta)\right|_{\zeta^{\perp}}\right)^{\dagger}=D f(\zeta)^{\dagger}$ is the minimum norm right inverse of $D f(\zeta)$. Thus

$$
\begin{aligned}
\gamma(f, \zeta) & =\max \left(1, \sup _{k \geq 2}\left\|\left(\left.D f(\zeta)\right|_{\zeta^{\perp}}\right)^{\dagger} \frac{D^{k} f(\zeta)}{k !}\right\|_{\zeta}^{\frac{1}{k-1}}\right) \\
& \leq \max \left(1, \sup _{k \geq 2}\left\|\left(\left.D f(\zeta)\right|_{x^{\perp}}\right)^{\dagger} \frac{D^{k} f(\zeta)}{k !}\right\|_{\zeta}^{\frac{1}{k-1}}\right)
\end{aligned}
$$

and by Lemma 6

$$
\leq \max \left(1, \sup _{k \geq 2}\left(c(x, \zeta) c(\zeta, x)^{k}\left\|\left(\left.D f(\zeta)\right|_{x^{\perp}}\right)^{\dagger} \frac{D^{k} f(\zeta)}{k !}\right\|_{x}\right)^{\frac{1}{k-1}}\right) .
$$

Moreover

$$
\left\|\left(\left.D f(\zeta)\right|_{x^{\perp}}\right)^{\dagger} \frac{D^{k} f(\zeta)}{k !}\right\|_{x} \leq\left\|\left(\left.D f(\zeta)\right|_{x^{\perp}}\right)^{\dagger} D f(x)\right\|_{x}\left\|\left(\left.D f(x)\right|_{x^{\perp}}\right)^{\dagger} \frac{D^{k} f(\zeta)}{k !}\right\|_{x} .
$$

Let us denote $v=\|x-\zeta\|_{x} \gamma(f, x)$. We have by Lemma $7 v \leq \sigma \alpha(f, x) \leq \sigma \alpha_{0}<$ $1-\sqrt{2} / 2$, and by Lemma 2

$$
\left\|\left(\left.D f(\zeta)\right|_{x^{\perp}}\right)^{\dagger} D f(x)\right\|_{x} \leq \frac{(1-v)^{2}}{\psi(v)} .
$$

We also have

$$
\begin{aligned}
\left\|\left(\left.D f(x)\right|_{x^{\perp}}\right)^{\dagger} \frac{D^{k} f(\zeta)}{k !}\right\|_{x} & \leq \sum_{l \geq 0}\left\|\left(\left.D f(x)\right|_{x^{\perp}}\right)^{\dagger} \frac{D^{k+l} f(x)}{k ! l !}\right\|_{x}\|x-\zeta\|_{x}^{l} \\
& \leq \sum_{l \geq 0}\left\|\frac{(k+l) !}{k ! l !} \gamma(f, x)^{k+l-1}\right\| x-\zeta \|_{x}^{l}=\frac{\gamma(f, x)^{k-1}}{(1-v)^{k+1}} .
\end{aligned}
$$

Since by Lemma $6 c(x, \zeta) \leq 1 /(1-v)$ and $c(\zeta, x) \leq 1+v$, we obtain

$$
\gamma(f, \zeta) \leq \max \left(1, \sup _{k \geq 2}\left(\frac{(1+v)^{k}}{1-v} \frac{(1-v)^{2}}{\psi(v)} \frac{\gamma(f, x)^{k-1}}{(1-v)^{k+1}}\right)^{\frac{1}{k-1}}\right) \leq\left(\frac{1+v}{1-v}\right)^{2} \frac{\gamma(f, x)}{\psi(v)}
$$

so that, by Lemmas 6 and 7

$$
\begin{aligned}
u_{\zeta} & =\|\zeta-y\|_{\zeta} \gamma(f, \zeta) \leq c(x, \zeta)\|\zeta-y\|_{x} \gamma(f, \zeta) \\
& \leq \frac{1}{1-v}(\sigma-1)\|x-y\|_{x}\left(\frac{1+v}{1-v}\right)^{2} \frac{\gamma(f, x)}{\psi(v)} \leq \tau u_{x}
\end{aligned}
$$

with

$$
\tau=\left.\frac{\sigma-1}{1-v}\left(\frac{1+v}{1-v}\right)^{2} \frac{1}{\psi(v)}\right|_{v=\sigma \alpha_{0}}
$$

since $v \leq \sigma \alpha_{0}$. From this expression we are able to deduce a numerical value for $\tau$. 
Lemma 9. With the same hypotheses as in Lemma 7 , there is a universal constant $0<\alpha_{1} \leq \alpha_{0}$ such that, if $\alpha(f, x) \leq \alpha_{1}$, then

$$
\beta(f, y) \leq \frac{2\left(1+\sigma \alpha_{0}\right)}{\psi\left(\alpha_{0}\right)} \beta(f, x)^{2} \gamma(f, x) .
$$

We can take $\alpha_{1}=0.009$.

Proof. We start from the following inequality:

$$
\begin{aligned}
\beta(f, y)= & \left\|\left(\left.D f(y)\right|_{y^{\perp}}\right)^{\dagger} f(y)\right\|_{y} \leq c(x, y)\left\|\left.\left(\left.D f(y)\right|_{y^{\perp}}\right)^{\dagger} D f(y)\right|_{x^{\perp}}\right\|_{x} \\
& \times\left\|\left(\left.D f(y)\right|_{x^{\perp}}\right)^{\dagger} D f(x)\right\|_{x}\left\|\left(\left.D f(x)\right|_{x^{\perp}}\right)^{\dagger} f(y)\right\|_{x}=\mathbf{1} \times \mathbf{2} \times \mathbf{3} \times \mathbf{4} .
\end{aligned}
$$

We have $\mathbf{1} \leq \frac{1}{1-u_{x}}$ by Lemma $6, \mathbf{3} \leq \frac{\left(1-u_{x}\right)^{2}}{\psi\left(u_{x}\right)}$ by Lemma 2 since $\left.D f(x)\right|_{x^{\perp}}$ is onto and $u_{x}=\alpha(f, x) \leq \alpha_{1}<1-\sqrt{2} / 2$. By Lemmas 5 and 6 we also have

$$
\mathbf{2} \leq c(\zeta, x)\left\|\left(\left.D f(y)\right|_{y^{\perp}}\right)^{\dagger} f(y)\right\|_{\zeta} \leq 2(1+v) \leq 2\left(1+\sigma \alpha_{0}\right)
$$

as in the proof of Lemma 8. This is accomplished when $d_{\zeta}\left(\operatorname{ker} D f(\zeta), W_{y}^{\perp}\right) \leq \sqrt{3} / 2$ and $\left.D f(y)\right|_{y^{\perp}}$ is onto (Lemma 5). The first inequality is satisfied when $u_{\zeta}<$ $1-\sqrt{2} / 2$ and $u_{\zeta} \phi\left(u_{\zeta}\right) \leq \sqrt{3} / 2$ (Lemma 4.b); Df $\left.(y)\right|_{y^{\perp}}$ is onto when $\frac{1}{\left(1-u_{\zeta}\right)^{2}}-$ $1+u_{\zeta}<1$. Since $u_{\zeta} \leq \tau u_{x}$ by Lemma 8 , these inequalities are satisfied when $\tau \alpha_{1}<1-\sqrt{2} / 2, \tau \alpha_{1} \phi\left(\tau \alpha_{1}\right) \leq \sqrt{3} / 2$ and $\frac{1}{\left(1-\tau \alpha_{1}\right)^{2}}-1+\tau \alpha_{1}<1$. This is accomplished with $\alpha_{1}=0.009$. Let us now give a bound for $\mathbf{4}$. We start from

$$
f(y)=f(x)+\sum_{k \geq 1} \frac{D^{k} f(x)}{k !}(y-x)^{k} .
$$

Since $y=N_{f}(x)$ we have $f(x)+D f(x)(y-x)=0$, so that

$$
\begin{aligned}
4=\left\|\left(\left.D f(x)\right|_{x^{\perp}}\right)^{\dagger} f(y)\right\|_{x} & \leq \sum_{k \geq 2}\left\|\left(\left.D f(x)\right|_{x^{\perp}}\right)^{\dagger} \frac{D^{k} f(x)}{k !}\right\|_{x}\|y-x\|_{x}^{k} \\
& \leq \frac{u_{x}}{1-u_{x}}\|y-x\|_{x} .
\end{aligned}
$$

Putting these inequalities togother gives the required result.

Lemma 10. With the same hypotheses as in Lemma 9,

$$
\gamma(f, y) \leq 2\left(\frac{1+\alpha_{0}}{1-\alpha_{0}}\right)^{2} \frac{1+\sigma \alpha_{0}}{\psi\left(\alpha_{0}\right)} \gamma(f, x) .
$$

Proof. Similarly to the proof of Lemma 9,

$$
\gamma(f, y)=\max \left(1, \sup _{k \geq 2}\left\|\left(\left.D f(y)\right|_{y^{\perp}}\right)^{\dagger} \frac{D^{k} f(y)}{k !}\right\|_{y}^{\frac{1}{k-1}}\right) .
$$

To bound this quantity in terms of $\gamma(f, x)$ we start from

$$
\begin{gathered}
\left\|\left(\left.D f(y)\right|_{y^{\perp}}\right)^{\dagger} \frac{D^{k} f(y)}{k !}\right\|_{y} \leq c(x, y) c(y, x)^{k} \times \mathbf{2} \times \mathbf{3} \times\left\|\left(\left.D f(x)\right|_{x^{\perp}}\right)^{\dagger} \frac{D^{k} f(y)}{k !}\right\|_{x} \\
\leq \frac{\left(1+u_{x}\right)^{k}}{1-u_{x}} 2\left(1+\sigma \alpha_{0}\right) \frac{\left(1-u_{x}\right)^{2}}{\psi\left(u_{x}\right)} \frac{\gamma(f, x)^{k-1}}{\left(1-u_{x}\right)^{k+1}} ;
\end{gathered}
$$


the last inequality may be proved as in Lemma 8 . This gives

$$
\gamma(f, y) \leq\left(\frac{1+u_{x}}{1-u_{x}}\right)^{2} \frac{2\left(1+\sigma \alpha_{0}\right)}{\psi\left(u_{x}\right)} \gamma(f, x),
$$

and we are done.

Proof of Theorem 1. We start from $x_{0}=x$ with $\left.D f(x)\right|_{x^{\perp}}$ onto and $\alpha(f, x) \leq \alpha_{u}=$ $1 / 137$. By Lemmas 9 and 10 and since $\alpha_{u} \leq \alpha_{1}$, we have $\alpha(f, y) \leq \tau_{1} \alpha(f, x)^{2}$, where

$$
\tau_{1}=\left(2 \frac{1+\alpha_{0}}{1-\alpha_{0}} \frac{1+\sigma \alpha_{0}}{\psi\left(\alpha_{0}\right)}\right)^{2} .
$$

We have $\tau_{1}=63.03684 \ldots$, so that $2 \tau_{1} \alpha(f, x) \leq 1$. We obtain, by induction over $k$,

$$
\alpha\left(f, x_{k}\right) \leq\left(\frac{1}{2}\right)^{2^{k}-1} \alpha(f, x) .
$$

Using Lemma 9 again, we get

$$
\beta(f, y) \leq \tau_{2} \alpha(f, x) \beta(f, x)
$$

with

$$
\tau_{2}=\frac{1+\sigma \alpha_{0}}{\psi\left(\alpha_{0}\right)}=6.00162 \ldots
$$

By induction over $k$ and since $2 \tau_{2} \alpha_{u} \leq 1$, we obtain

$$
\left\|x_{k+1}-x_{k}\right\|_{x_{k}} \leq \beta\left(f, x_{k}\right) \leq\left(\frac{1}{2}\right)^{2^{k}-1} \beta(f, x) .
$$

We now notice that

$$
d_{R}\left(x_{k+1}, x_{k}\right) \leq\left\|x_{k+1}-x_{k}\right\|_{x_{k}},
$$

because $x_{k+1}-x_{k} \in T_{x_{k}} \Pi_{i} \mathbb{P}\left(\mathbb{E}_{i}\right)$. The rest of the proof is easy.

2.3. $\gamma$-theorem. In this section we give a proof of Theorem 2. According to Theorem 1, $x$ will be an approximate zero if $\left.D f(x)\right|_{x^{\perp}}$ is onto and $\alpha(f, x) \leq \alpha_{u}$. Let us denote $w=\|x-\zeta\|_{\zeta} \gamma(f, \zeta)$. By Lemma 3.c, $\left.D f(x)\right|_{x^{\perp}}$ is onto when $\frac{1}{(1-w)^{2}}-$ $1+w<1$. This is accomplished when $w \leq 0.24512 \ldots$ Let us now compute a bound for $\alpha(f, x)$. We have by Lemma 6

$$
\begin{gathered}
\beta(f, x)=\left\|\left(\left.D f(x)\right|_{x^{\perp}}\right)^{\dagger} f(x)\right\|_{x} \leq \frac{1}{1-w}\left\|\left(\left.D f(x)\right|_{x^{\perp}}\right)^{\dagger} f(x)\right\|_{\zeta} \\
\leq \frac{1}{1-w}\left\|\left(\left.D f(x)\right|_{x^{\perp}}\right)^{\dagger} D f(\zeta)\right\|_{\zeta}\left\|\left(\left.D f(\zeta)\right|_{\zeta^{\perp}}\right)^{\dagger} f(x)\right\|_{\zeta}=\mathbf{1} \times \mathbf{2} \times \mathbf{3} .
\end{gathered}
$$

By Lemma 2 we get $2 \leq \frac{(1-w)^{2}}{\psi(w)}$, since $w<1-\sqrt{2} / 2$. Moreover,

$$
f(x)=D f(\zeta)(x-\zeta)+\sum_{k \geq 2} \frac{D^{k} f(\zeta)}{k !}(x-\zeta),
$$

so that $\mathbf{3} \leq \frac{\|x-\zeta\|_{\zeta}}{1-w}$. This gives

$$
\beta(f, x) \leq \frac{\|x-\zeta\|_{\zeta}}{\psi(w)} .
$$


To get a bound for $\gamma(f, x)$ we use an argument similar to Lemma 8 , and we obtain

$$
\gamma(f, x) \leq \frac{(1+w)^{2}}{(1-w)^{2} \psi(w)} \gamma(f, \zeta)
$$

Thus

$$
\alpha(f, x) \leq\left(\frac{(1+w)}{(1-w) \psi(w)}\right)^{2} \gamma(f, \zeta)\|x-\zeta\|_{\zeta}
$$

This quantity is $\leq \alpha_{u}$ when

$$
\gamma_{u} \leq \alpha_{u}\left(\frac{(1+w)}{(1-w) \psi(w)}\right)^{-2} .
$$

According to the bound $w \leq 0.24512 \ldots$ and the value $\alpha_{u}=1 / 137$ we can take $\gamma_{u}=0.00005 \ldots$, but such a value is pessimistic. A better bound might be obtained by a direct proof of this $\gamma$-theorem. According to Theorem 1 there is a zero $\zeta^{\prime}$ of $f$ such that the Newton sequence $x_{k}$ converges to $\zeta^{\prime}$ and

$$
d_{R}\left(\zeta^{\prime}, x_{k}\right) \leq \sigma\left(\frac{1}{2}\right)^{2^{k}-1} \beta(f, x) .
$$

With $k=1$ and by the previous estimation for $\beta$ we obtain

$$
d_{R}\left(\zeta^{\prime}, x\right) \leq \frac{\sigma}{\psi(w)}\|x-\zeta\|_{\zeta} \leq 2.86543 \ldots\|x-\zeta\|_{\zeta} \leq 3\|x-\zeta\|_{\zeta}
$$

As has been proved before, we have $\psi\left(\gamma_{u}\right) \beta(f, x) \leq\|x-\zeta\|_{\zeta}$. We also have noticed that $\alpha(f, x) \leq \alpha_{u}$, so that, by Theorem 1 ,

$$
d_{R}\left(\zeta^{\prime}, x_{k}\right) \leq \sigma\left(\frac{1}{2}\right)^{2^{k}-1} \beta(f, x)
$$

for some root $\zeta^{\prime}$ of $f$. This inequality also applies with $N_{f}(x)$ instead of $x$ and gives, using Lemma 9 ,

$$
d_{R}\left(\zeta^{\prime}, N_{f}(x)\right) \leq \sigma \beta(f, y) \leq c_{1} \beta(f, x)^{2} \gamma(f, x)
$$

We now use the estimate $\gamma(f, x) \leq c_{2} \gamma(f . x)$ obtained previously to obtain

$$
d_{R}\left(\zeta^{\prime}, N_{f}(x)\right) \leq c_{u} \gamma(f, \zeta)\|\zeta-x\|_{\zeta}^{2} .
$$

Proof of Corollary 1. Let us first remark that $d_{R}(x, y) \leq\|x-y\|_{x}$ and say that $\frac{5}{6}\|x-y\|_{x} \leq d_{R}(x, y)$ if we take representatives such that $x-y \in x^{\perp}$ and if $d_{R}(x, y)$ is small enough. This property is supposed to be satisfied when

$$
d_{R}(x, y) \leq d_{R}(x, y) \gamma(f, \zeta) \leq \delta_{u} .
$$

If we take representatives of $x$ and $\zeta$ satisfying $\zeta-x \in \zeta^{\perp}$, we obtain

$$
\|\zeta-x\|_{\zeta} \gamma(f, \zeta) \leq \frac{6}{5} \delta_{u} \leq \gamma_{u}
$$

so that Theorem 2 gives a Newton's sequence converging to a zero $\zeta^{\prime}$ of $f$ with

$$
d_{R}\left(\zeta^{\prime}, x_{k}\right) \leq \sigma\left(\frac{1}{2}\right)^{2^{k}-1} \beta(f, x)
$$


This inequality also applies with $N_{f}(x)$ instead of $x$, and gives

$$
d_{R}\left(\zeta^{\prime}, x_{k+1}\right) \leq \sigma\left(\frac{1}{2}\right)^{2^{k}-1} \beta\left(f, N_{f}(x)\right) .
$$

We now use Lemma 9 to bound $\beta\left(f, N_{f}(x)\right)$ in terms of $\beta(f, x)$. Then we use the estimates of $\gamma(f, x)$ in terms of $\gamma(f, \zeta)$ and $\beta(f, x)$ in terms of $\|x-\zeta\|_{\zeta}$ obtained in the proof of Theorem 2 to obtain

$$
\begin{gathered}
d_{R}\left(\zeta^{\prime}, x_{k+1}\right) \leq c_{1}\left(\frac{1}{2}\right)^{2^{k}-1} \beta(f, x)^{2} \gamma(f, x) \leq c_{2}\left(\frac{1}{2}\right)^{2^{k}-1} \gamma(f, \zeta)\|x-\zeta\|_{\zeta}^{2} \\
\leq c_{3} \frac{6}{5} \delta_{u}\left(\frac{1}{2}\right)^{2^{k}-1}\|x-\zeta\|_{\zeta} \leq\left(\frac{1}{2}\right)^{2^{k}} d_{R}(x-\zeta)
\end{gathered}
$$

if $c_{3} \frac{72}{25} \delta_{u} \leq 1$.

2.4. Newton's method for the evaluation map. In this section we give the proofs of Theorems 3 and 4 . We first describe Newton's iterate $N_{e v}(f, x)$. In a first lemma we study the derivative of the evaluation map.

Lemma 11. For any $(f, x) \in \mathcal{H}_{(d)} \times \mathbb{C}^{n}$ we have

$$
\operatorname{Dev}(f, x)(\dot{f}, \dot{x})=\dot{f}(x)+D f(x) \dot{x} .
$$

Moreover, $\operatorname{Dev}(f, x)$ is always onto.

The following representation lemma will be useful later. A proof may be found in Blum-Cucker-Shub-Smale [2], Section 14.1.

Lemma 12. For any $f_{i} \in \mathcal{H}_{d_{i}}$ and $x \in \mathbb{C}^{n}$ we have

$$
f_{i}(x)=\left\langle f_{i}(z),\langle z, x\rangle^{d_{i}}\right\rangle, \quad\left\|\langle z, x\rangle^{d_{i}}\right\|=\|x\|^{d_{i}}
$$

and

$$
\mid f_{i}(x) \leq\left\|f_{i}\right\|\|x\|^{d_{i}} .
$$

Let us denote by $f^{\perp}$ and $x^{\perp}$ the vector subspaces in $\mathcal{H}_{(d)}$ and $\mathbb{C}^{n}$ that are orthogonal complements of $\mathbb{C} f$ and $\mathbb{C} x$. In the sequel we suppose that $\|f\|=$ $\|x\|=1$. In such a case, the Hermitian structure \|\|$_{(f, x)}$ coincides with the usual product structure on $\mathcal{H}_{(d)} \times \mathbb{C}^{n}$. Newton's iterate for eval is given by

$$
N_{e v}(f, x)=(f, x)-\left(\left.\operatorname{Dev}(f, x)\right|_{f^{\perp} \times x^{\perp}}\right)^{\dagger} f(x) .
$$

In the following lemma we compute this Moore-Penrose inverse. We first have to introduce some notations. $f(x)$ is a column vector, $D f_{i}(x)$ is a row vector with entries $\frac{\partial f_{i}}{\partial z_{j}}(x), 1 \leq j \leq n$, and $D f(x)$ is the $m \times n$ matrix with rows $D f_{i}(x)$, $1 \leq i \leq m$. For any matrix $A$ we denote by $A^{\star}$ its adjoint. With these notations, the usual scalar product in $\mathbb{C}^{n}$ is given by $\langle x, y\rangle=y^{\star} x$, and the value at $\dot{x}$ of the derivative $D f(x)$ is also the product $D f(x) \dot{x}$ of the matrix $D f(x)$ by the column vector $\dot{x}$.

Lemma 13. For any $\mu \in \mathbb{C}^{m}$ we have $\left(\left.\operatorname{Dev}(f, x)\right|_{f^{\perp} \times x^{\perp}}\right)^{\dagger} \mu=(\dot{f}, \dot{x})$ with

$$
\dot{f}_{j}(z)=\lambda_{j}\langle z, x\rangle^{d_{j}}-\left(\sum_{i=1}^{m} \lambda_{i} \overline{f_{i}(x)}\right) f_{j}(z),
$$




$$
\begin{gathered}
\dot{x}=D f(x)^{\star} \lambda-\left\langle D f(x)^{\star} \lambda, x\right\rangle x, \\
\lambda=\left(I_{m}+D f(x) D f(x)^{\star}-f(x) f(x)^{\star}-\mathcal{B} f(x) f(x)^{\star} \mathcal{B}\right)^{-1} \mu,
\end{gathered}
$$

where $I_{m}$ is the identity matrix and $\mathcal{B}$ the diagonal matrix with entries $d_{i}, 1 \leq i \leq$ $m$.

Proof. We have $(\dot{f}, \dot{x}) \in \operatorname{ker} \operatorname{Dev}(f, x)$ if and only if $\dot{f}(x)+D f(x) \dot{x}=0$ or, according to Lemma 12,

$$
\left\langle\dot{f}_{i}(z),\langle z, x\rangle^{d_{i}}\right\rangle+\left\langle\dot{x}, D f_{i}(x)^{\star}\right\rangle=0, \quad 1 \leq i \leq m,
$$

or, in terms of the $\mathcal{H}_{(d)}$, scalar product,

$$
\left\langle\dot{f}(z),\left(0, \ldots,\langle z, x\rangle^{d_{i}}, \ldots, 0\right)\right\rangle+\left\langle\dot{x}, D f_{i}(x)^{\star}\right\rangle=0, \quad 1 \leq i \leq m .
$$

Thus ker $\operatorname{Dev}(f, x)^{\perp}$ consists in the $(m+1)$-tuples

$$
\left(\lambda_{1}\langle z, x\rangle^{d_{1}}, \ldots, \lambda_{m}\langle z, x\rangle^{d_{m}}, D f(x)^{\star} \lambda\right), \quad \lambda \in \mathbb{C}^{m} .
$$

In fact $\left(\left.\operatorname{Dev}(f, x)\right|_{f^{\perp \times} \times x^{\perp}}\right)^{\dagger}$ involves the orthogonal complement in $f^{\perp} \times x^{\perp}$ of ker $\left.\operatorname{Dev}(f, x)\right|_{f^{\perp} \times x^{\perp}}$. This subspace is equal to

$$
\begin{aligned}
& \left(\text { ker } \operatorname{Dev}(f, x) \cap\left(f^{\perp} \times x^{\perp}\right)\right)^{\perp} \cap\left(f^{\perp} \times x^{\perp}\right) \\
& \quad=\left(\operatorname{ker} \operatorname{Dev}(f, x)^{\perp}+(\mathbb{C} f \times \mathbb{C} x)\right) \cap\left(f^{\perp} \times x^{\perp}\right) .
\end{aligned}
$$

Any element in this subspace is equal to some

$$
(\dot{f}, \dot{x})=\left(\alpha f_{1}+\lambda_{1}\langle z, x\rangle^{d_{1}}, \ldots, \alpha f_{m}+\lambda_{m}\langle z, x\rangle^{d_{m}}, \beta x+D f(x)^{\star} \lambda\right)
$$

with $\langle\dot{f}, f\rangle=0$ and $\langle\dot{x}, x\rangle=0$. Consequently

$$
\alpha=-\sum_{i} \lambda_{i} \overline{f_{i}(x)} \text { and } \beta=-\left\langle D f(x)^{\star} \lambda, x\right\rangle,
$$

since $\|f\|=\|x\|=1$. Let us now compute

$$
\left(\left.\operatorname{Dev}(f, x)\right|_{f^{\perp} \times x^{\perp}}\right)^{\dagger} \mu=(\dot{f}, \dot{x}) .
$$

We have $(\dot{f}, \dot{x})$ as before and $\dot{f}(x)+D f(x) \dot{x}=\mu$, so that

$$
\left(I_{m}+D f(x) D f(x)^{\star}-f(x) f(x)^{\star}-\mathcal{B} f(x) f(x)^{\star} \mathcal{B}\right) \lambda=\mu,
$$

using Euler's formula for $f_{i}$ i.e $D f_{i}(x) x=d_{i} f_{i}(x)$. The invertibility of this matrix is a consequence of the invertibility of the restriction of $\left.\operatorname{Dev}(f, x)\right|_{f^{\perp} \times x^{\perp}}$ to the orthogonal complement in $f^{\perp} \times x^{\perp}$ of ker $\left.\operatorname{Dev}(f, x)\right|_{f^{\perp} \times x^{\perp}}$.

Lemma 14. For any $x \in \mathbb{C}^{n}$ with $\|x\|=1, \dot{x}_{1}, \ldots, \dot{x}_{k} \in \mathbb{C}^{n}$, we have

$$
\left|D^{k} f_{i}(x)\left(\dot{x}_{1}, \ldots, \dot{x}_{k}\right)\right| \leq d_{i}\left(d_{i}-1\right) \ldots\left(d_{i}-k+1\right)\left\|f_{i}\right\|\left\|\dot{x}_{1}\right\| \ldots\left\|\dot{x}_{k}\right\| .
$$

The proof of this lemma may be found in Blum-Cucker-Shub-Smale [2], Section 14.1.

\section{Lemma 15.}

$$
\begin{aligned}
D^{k} e v & \left(f_{i}, x\right)\left(\dot{f}_{1}, \dot{x}_{1}, \ldots, \dot{f}_{k}, \dot{x}_{k}\right) \\
& =D^{k} f_{i}(x)\left(\dot{x}_{1}, \ldots, \dot{x}_{k}\right)+\sum_{j=1}^{k} D^{k-1} \dot{f}_{j}(x)\left(\dot{x}_{1}, \ldots, \underline{\dot{x}_{j}}, \ldots, \dot{x}_{k}\right),
\end{aligned}
$$

where ${\underline{\dot{x}_{j}}}_{\text {indicates that }} \dot{x}_{j}$ is missing. 
Lemma 16.

$$
\frac{\left\|D^{k} e v\left(f_{i}, x\right)\right\|}{k !} \leq\left(\begin{array}{c}
d_{i} \\
k
\end{array}\right)\left\|f_{i}\right\|+\left(\begin{array}{c}
d_{i} \\
k-1
\end{array}\right) .
$$

The proof of this lemma is an easy consequence of Lemmas 14 and 15.

Lemma 17. When $f(x)=0$, then

$$
\left\|\left(\left.\operatorname{Dev}(f, x)\right|_{f^{\perp} \times x^{\perp}}\right)^{\dagger}\right\|=\left\|\left(I+D f(x) D f(x)^{\star}\right)^{-1}\right\| \leq 1 .
$$

Proof. When $f(x)=0$ we have by Lemma 13

$$
\left(\left.\operatorname{Dev}(f, x)\right|_{f^{\perp} \times x^{\perp}}\right)^{\dagger} \mu=(\dot{f}, \dot{x})
$$

with

$$
\dot{f}_{i}(z)=\lambda_{i}\langle z, x\rangle^{d_{i}}, \quad \dot{x}=D f(x)^{\star} \lambda \text { and } \lambda=\left(I+D f(x) D f(x)^{\star}\right)^{-1} \mu .
$$

Thus

$$
\left\|\left(\left.\operatorname{Dev}(f, x)\right|_{f^{\perp} \times x^{\perp}}\right)^{\dagger}\right\|^{2}=\max \|\lambda\|^{2}+\left\|D f(x)^{\star} \lambda\right\|^{2},
$$

where the maximum is taken for $\|\mu\|=1$. This maximum is also equal to

$$
\begin{aligned}
\max \lambda^{\star}\left(I+D f(x) D f(x)^{\star}\right) \lambda & =\max \mu^{\star}\left(I+D f(x) D f(x)^{\star}\right)^{-1} \mu \\
& =\left\|\left(I+D f(x) D f(x)^{\star}\right)^{-1}\right\| .
\end{aligned}
$$

This quantity is always less than or equal to 1 , since adding to the identity matrix a psd matrix increases the eigenvalues.

Proof of Theorem 3. For any $(f, x) \in V$ we have

$$
\left\|\left(\left.\operatorname{Dev}(f, x)\right|_{f^{\perp} \times x^{\perp}}\right)^{\dagger} \frac{D^{k} e v(f, x)}{k !}\right\| \leq\left\|\left(\left.\operatorname{Dev}(f, x)\right|_{f^{\perp} \times x^{\perp}}\right)^{\dagger}\right\|\left\|\frac{D^{k} e v(f, x)}{k !}\right\| .
$$

By Lemma 17 the first term is bounded by 1, and by Lemma 16 we get

$$
\leq\left(\sum_{i=1}^{m}\left(\left(\begin{array}{c}
d_{i} \\
k
\end{array}\right)\left\|f_{i}\right\|+\left(\begin{array}{c}
d_{i} \\
k-1
\end{array}\right)\right)^{2}\right)^{1 / 2} \leq\left(\begin{array}{c}
D \\
k
\end{array}\right)(1+\sqrt{m}) .
$$

We now have to take the $(k-1)$-th root of this expression and its maximum for $k \geq 2$. The well known inequality

$$
\left(\begin{array}{l}
D \\
k
\end{array}\right)^{\frac{1}{k-1}} \leq\left(\begin{array}{l}
D \\
2
\end{array}\right), \quad k \geq 2
$$

gives the required result.

Proof of Theorem 4. This theorem is a consequence of Theorem 2, Corollary 1 and Theorem 3 . 
2.5. Path-following. In this section we give the proof of Theorem 5 . We choose the subdivision $t_{i}, 0 \leq i \leq p$, such that all the distances $d_{R}\left(\zeta_{t_{i+1}}, \zeta_{t_{i}}\right)$ are equal. Then we have

$$
p d_{R}\left(\zeta_{t_{i+1}}, \zeta_{t_{i}}\right)=\sum_{i} d_{R}\left(\zeta_{t_{i+1}}, \zeta_{t_{i}}\right) \leq L_{\zeta}
$$

with $L_{\zeta}$ the length of the curve $t \in[0,1] \rightarrow \zeta_{t}$ in the Riemannian distance

$$
L_{\zeta}=\int_{0}^{1}\left\|\dot{\zeta}_{t}\right\|_{\zeta_{t}} d t
$$

and $\dot{\zeta}_{t}$ the derivative with respect to $t$. Since

$$
\dot{\zeta}_{t}=\frac{d \zeta_{t}}{d f_{t}} \frac{d f_{t}}{d t} \quad \text { and } \quad\left\|\frac{d \zeta_{t}}{d f_{t}}\right\|_{\zeta_{t}} \leq \mu
$$

we obtain $L_{\zeta} \leq \mu L$, so that

$$
d_{R}\left(\zeta_{t_{i+1}}, \zeta_{t_{i}}\right) \gamma \leq \frac{\gamma \mu L}{p} \leq \frac{\delta_{u}}{2}
$$

We have to prove that

$$
d_{R}\left(x_{i}, \zeta_{t_{i}}\right) \gamma\left(f_{t_{i}}, \zeta_{t_{i}}\right) \leq \delta_{u}
$$

by Corollary 1 . This will be accomplished if

$$
d_{R}\left(x_{i}, \zeta_{t_{i}}\right) \gamma \leq \frac{\delta_{u}}{2}
$$

We prove this inequality by induction over $i$. The case $i=0$ is easy, since $\zeta_{0}=x_{0}$. We have, by Lemma 6 ,

$$
d_{R}\left(x_{i}, \zeta_{t_{i+1}}\right) \gamma \leq d_{R}\left(\zeta_{t_{i+1}}, \zeta_{t_{i}}\right)+d_{R}\left(\zeta_{t_{i}}, x_{i}\right) \leq \delta_{u} .
$$

By Corollary 1 this gives

$$
d_{R}\left(x_{i+1}, \zeta_{t_{i+1}}\right)=d_{R}\left(N_{f_{i+1}}\left(x_{i}\right), \zeta_{t_{i+1}}\right) \leq d_{R}\left(x_{i}, \zeta_{t_{i+1}}\right) / 2 \leq \delta_{u} / 2,
$$

and we are done.

2.6. Newton's method for the generalized eigenvalue problem. In this section we first give a precise description of multihomogeneous Newton's iterate for the generalized eigenvalue problem (gep); then we compute its condition number and we prove Theorem 6 . We have introduced previously

$$
F_{(A, B)}: \mathbb{C}^{2} \times \mathbb{C}^{n} \rightarrow \mathbb{C}^{n}, \quad F_{(A, B)}(\alpha, \beta, x)=(\beta A-\alpha B) x,
$$

whose zeros are the eigenpairs of $(A, B)$. Multihomogeneous Newton's iterate is equal to

$$
N_{F_{(A, B)}}(\alpha, \beta, x)=(\alpha, \beta, x)-\left.D F_{(A, B)}(\alpha, \beta, x)\right|_{(\alpha, \beta, x)^{\perp}} ^{\dagger}(\beta A-\alpha B) x .
$$

This iterate is computed in the next proposition 
Proposition 5. Let $(A, B)$ be a regular matrix pair, $(\alpha, \beta) \in \mathbb{C}^{2}$ and $x \in \mathbb{C}^{n}$ both non-zero. If $(\alpha, \beta)$ is not an eigenvalue of the pair $(A, B)$, then multihomogeneous Newton's iterate is given by

$$
\begin{gathered}
N_{F_{(A, B)}}(\alpha, \beta, x)=(\alpha+\dot{\lambda} \bar{\beta}, \beta-\dot{\lambda} \bar{\alpha}, x-\dot{x}), \\
\dot{\lambda}=\frac{\langle x, x\rangle}{\left\langle(\beta A-\alpha B)^{-1}(\bar{\alpha} A+\bar{\beta} B) x, x\right\rangle}, \\
\dot{x}=(\beta A-\alpha B)^{-1}((\beta-\dot{\lambda} \bar{\alpha}) A-(\alpha+\dot{\lambda} \bar{\beta}) B) x .
\end{gathered}
$$

Proof. The subspace $(\alpha, \beta, x)^{\perp}$ consists in those couples $(\dot{\alpha}, \dot{\beta}, \dot{x}) \in \mathbb{C}^{2} \times \mathbb{C}^{n}$ satisfying $\langle\dot{x}, x\rangle=0$ and $(\dot{\alpha}, \dot{\beta})=\dot{\lambda}(-\bar{\beta}, \bar{\alpha})$. Thus $(\alpha, \beta, x)^{\perp}$ has dimension $n$. We also have

$$
D F_{(A, B)}(\alpha, \beta, x)(\dot{\alpha}, \dot{\beta}, \dot{x})=(\dot{\beta} A-\dot{\alpha} B) x+(\beta A-\alpha B) \dot{x} .
$$

To compute Newton's iterate we have to solve the following system:

$$
\begin{gathered}
(\dot{\beta} A-\dot{\alpha} B) x+(\beta A-\alpha B) \dot{x}=(\beta A-\alpha B) x, \\
(\dot{\alpha}, \dot{\beta})=\dot{\lambda}(-\bar{\beta}, \bar{\alpha}) \text { and }\langle\dot{x}, x\rangle=0 .
\end{gathered}
$$

Since $(\alpha, \beta)$ is not an eigenvalue, the matrix $(\beta A-\alpha B)$ is nonsingular. Multiplying the first equation by $(\beta A-\alpha B)^{-1}$ and then taking the scalar product with $x$ gives

$$
\dot{\lambda}\left\langle(\beta A-\alpha B)^{-1}(\bar{\alpha} A+\bar{\beta} B) x, x\right\rangle=\langle x, x\rangle,
$$

and we are done.

We now compute the condition number for the gep. According to Definition 4, when $(\alpha, \beta, x)$ is an eigenpair of $(A, B)$ then

$$
\mu(A, B, \alpha, \beta, x)=\left\|\left.D F_{(A, B)}(\alpha, \beta, x)\right|_{(\alpha, \beta, x)^{\perp}} ^{\dagger}\right\|_{(\alpha, \beta, x)} .
$$

In Dedieu $[3$ a similar computation is given, but the condition number of the eigenvalue and the condition number for the eigenvector are computed separately. We prove here that the condition number for the eigenpair is equal to the maximum of 1 and the condition number for the eigenvector.

Proposition 6. Let $(A, B)$ be a regular matrix pair, $(\alpha, \beta) \in \mathbb{C}^{2}$ and $x \in \mathbb{C}^{n}$ both non-zero with

$$
(\beta A-\alpha B) x=0 \text { and } A x \neq 0 .
$$

Suppose that $(\alpha, \beta)$ is a simple eigenvalue of the pair $(A, B)$, i.e., a simple root of the polynomial $\operatorname{det}(\beta A-\alpha B)$. Then

$$
\mu(A, B, \alpha, \beta, x)=\max \left(1,\left\|\left(\left.\Pi_{(A x)^{\perp}}(\beta A-\alpha B)\right|_{x^{\perp}}\right)^{-1}\right\|\left(|\alpha|^{2}+|\beta|^{2}\right)^{1 / 2}\right)
$$

with $\Pi_{(A x) \perp}$ the orthogonal projection over $(A x)^{\perp}$.

Before proving this proposition we make some comments.

1. For an eigenpair $(\alpha, \beta, x)$ of $(A, B)$ we always have $A x \neq 0$ or $B x \neq 0$, since the pair $(A, B)$ is regular. When both are nonzero then $(A x)^{\perp}=(B x)^{\perp}$, since $\beta A x=\alpha B x$. 
2. $\mu(A, B, \alpha, \beta, x)$ is invariant under scaling on both the eigenvector and the eigenvalue. It is, in fact, defined on the variety

$$
\mathcal{V}=\left\{(A, B, \alpha, \beta, x) \in \mathcal{M}_{n}(\mathbb{C})^{2} \times \mathbb{P}\left(\mathbb{C}^{2}\right) \times \mathbb{P}\left(\mathbb{C}^{n}\right):(\beta A-\alpha B) x=0\right\} .
$$

This is a consequence of Lemma 1 and the definition of $\mu$.

3. The condition number is invariant under unitary transformations:

$$
\mu(A, B, \alpha, \beta, x)=\mu\left(V^{\star} A U, V^{\star} B U, \alpha, \beta, U^{\star} x\right)
$$

for any unitary matrices $U$ and $V$. We do not prove this fact here; it is a consequence of the definition of $\mu$, of the chain rule and the unitary invariance of the spectral norm for matrices.

4. The linear operator $\left.\Pi_{(A x)^{\perp}}(\beta A-\alpha B)\right|_{x^{\perp}}$ is nonsingular if and only if $(\alpha, \beta)$ is a simple eigenvalue of the pair $(A, B)$. See Dedieu [3], Lemma 4.1, for a proof of this fact.

Proof of Proposition 6. By the invariance property under scaling we can suppose that $|\alpha|^{2}+|\beta|^{2}=1$ and $\|x\|=1$. By the unitary invariance property, using the Schur decomposition for a matrix pair (see Dedieu [3] or Stewart-Sun [15], Chap. 6, Theorem 1.9) we may suppose that $x=e_{1}$, the first basis vector in $\mathbb{C}^{n}$, and

$$
A=\left(\begin{array}{cc}
\alpha & a^{\star} \\
0 & \tilde{A}
\end{array}\right) \text { and } B=\left(\begin{array}{cc}
\beta & b^{\star} \\
0 & \tilde{B}
\end{array}\right)
$$

We have

$$
D F_{(A, B)}(\alpha, \beta, x)(\dot{\alpha}, \dot{\beta}, \dot{x})=(\dot{\beta} A-\dot{\alpha} B) x+(\beta A-\alpha B) \dot{x}
$$

When $(\dot{\alpha}, \dot{\beta}) \perp(\alpha, \beta)$ and $\dot{x} \perp x$ we can write

$$
(\dot{\alpha}, \dot{\beta})=\dot{\lambda}(-\bar{\beta}, \bar{\alpha}) \text { and } \dot{x}=\left(\begin{array}{c}
0 \\
\dot{y}
\end{array}\right)
$$

so that

$$
D F_{(A, B)}(\alpha, \beta, x)(\dot{\alpha}, \dot{\beta}, \dot{x})=\left(\begin{array}{c}
\dot{\lambda} \\
(\beta \tilde{A}-\alpha \tilde{B}) \dot{y}
\end{array}\right)=\left(\begin{array}{cc}
1 & 0 \\
0 & \beta \tilde{A}-\alpha \tilde{B}
\end{array}\right)\left(\begin{array}{l}
\dot{\lambda} \\
\dot{y}
\end{array}\right) .
$$

The condition number is equal to

$$
\left\|\left.D F_{(A, B)}(\alpha, \beta, x)\right|_{(\alpha, \beta, x) \perp} ^{-1}\right\|_{(\alpha, \beta, x)} .
$$

Since $|\alpha|^{2}+|\beta|^{2}=1$ and $\|x\|=1$, the endomorphism norm involved in this definition is the usual spectral norm, so that

$$
\mu(A, B, \alpha, \beta, x)=\max \left(1,\left\|(\beta \tilde{A}-\alpha \tilde{B})^{-1}\right\|\right) .
$$

To conclude we just notice that

$$
\beta \tilde{A}-\alpha \tilde{B}=\left.\Pi_{(A x)^{\perp}}(\beta A-\alpha B)\right|_{x^{\perp}} .
$$

We now give an estimate for $\gamma\left(F_{(A, B)}, \alpha, \beta, x\right)$ when $(\alpha, \beta)$ is a simple eigenvalue of the pair $(A, B)$.

Proposition 7. Under the hypothesis above

$$
\gamma\left(F_{(A, B)}, \alpha, \beta, x\right) \leq \max \left(1, \frac{1}{2} \mu(A, B, \alpha, \beta, x)\left(\|A\|^{2}+\|B\|^{2}\right)^{1 / 2}\right) .
$$


Proof. We first suppose that $|\alpha|^{2}+|\beta|^{2}=1$ and $\|x\|=1$, since, by Proposition 2 , $\gamma$ is invariant under scaling. We have

$$
D^{2} F(\alpha, \beta, x)\left(\dot{\alpha}_{1}, \dot{\beta}_{1}, \dot{x}_{1}\right)\left(\dot{\alpha}_{2}, \dot{\beta}_{2}, \dot{x}_{2}\right)=\left(\dot{\beta}_{2} A-\dot{\alpha}_{2} B\right) \dot{x}_{1}+\left(\dot{\beta}_{1} A-\dot{\alpha}_{1} B\right) \dot{x}_{2},
$$

so that, when $\left(\dot{\alpha}_{i}, \dot{\beta}_{i}\right)=\dot{\lambda}_{i}(-\bar{\beta}, \bar{\alpha}), i=1,2$,

$$
=(\bar{\alpha} A+\bar{\beta} B)\left(\dot{\lambda}_{2} \dot{x}_{1}+\dot{\lambda}_{1} \dot{x}_{2}\right) .
$$

Since $(\alpha, \beta)$ and $x$ are normalized, we have

$$
\left\|D^{2} F(\alpha, \beta, x)\right\|_{(\alpha, \beta, x)}=\max _{\left|\dot{\lambda}_{i}\right|^{2}+\left\|\dot{x}_{i}\right\|^{2}=1}\left\|(\bar{\alpha} A+\bar{\beta} B)\left(\dot{\lambda}_{2} \dot{x}_{1}+\dot{\lambda}_{1} \dot{x}_{2}\right)\right\|=\|\bar{\alpha} A+\bar{\beta} B\|,
$$

because

$$
\left\|\dot{\lambda}_{2} \dot{x}_{1}+\dot{\lambda}_{1} \dot{x}_{2}\right\| \leq\left|\dot{\lambda}_{2}\right|\left\|\dot{x}_{1}\right\|+\left|\dot{\lambda}_{1}\right|\left\|\dot{x}_{2}\right\| \leq\left(\left|\dot{\lambda}_{1}\right|^{2}+\left\|\dot{x}_{1}\right\|^{2}\right)^{1 / 2}\left(\left|\dot{\lambda}_{2}\right|^{2}+\left\|\dot{x}_{2}\right\|^{2}\right)^{1 / 2}=1 .
$$

This gives

$$
\begin{aligned}
& \gamma\left(F_{(A, B)}, \alpha, \beta, x\right)=\max \left(1, \frac{1}{2} \|\left(\left.D F(\alpha, \beta, x)\right|_{(\alpha, \beta, x)^{\perp}} ^{-1} D^{2} F(\alpha, \beta, x) \|_{(\alpha, \beta, x)}\right)\right. \\
& \quad \leq \max \left(1, \frac{1}{2} \mu(A, B, \alpha, \beta, x)\|\bar{\alpha} A+\bar{\beta} B\|\right) \\
& \left.\quad \leq \max \left(1, \frac{1}{2} \mu(A, B, \alpha, \beta, x)\left(\|A\|^{2}+\|B\|^{2}\right)^{1 / 2}\right)\right) .
\end{aligned}
$$

Proof of Theorem 6. We put together Theorem 5, Proposition 6 and Proposition 7 to obtain the required estimate.

\section{REFERENCES}

[1] E. Allgower, K. Georg, Numerical Continuation Methods, Springer Verlag (1990). MR 92a:65165

[2] L. Blum, F. Cucker, M. Shub, S. Smale, Complexity and Real Computation, Springer Verlag (1997). MR 99a:68070

[3] J. P. Dedieu, Condition Operators, Condition Numbers, and Condition Number Theorem for the Generalized Eigenvalue Problem, Linear Algebra and its Applications 263, 1-24 (1997). MR 98e: 15008

[4] J. P. Dedieu, Condition Number Analysis for Sparse Polynomial Systems, in : Foundations of Computational Mathematics, F. Cucker, M. Shub Eds. Springer (1997).

[5] T. Y. Li, Numerical Solution of Multivariate Polynomial Systems by Homotopy Continuation Methods, Acta Numerica 6, 399-436 (1997). CMP 98:06

[6] G. Malajovich, On Generalized Newton Algorithms, Theoretical Computer Science 133, 65-84 (1994). MR 95g:65073

[7] H. Royden. Newton's Method. Preprint.

[8] M. Shub, Some Remarks on Bézout's Theorem and Complexity, in : Proceedings of the Smalefest, M. V. Hirsch, J. E. Marsden, M. Shub Eds., Springer, 443-455 (1993). MR 95a:14002

[9] M. Shub, S. Smale, Complexity of Bézout's Theorem I : Geometric Aspects, J. Am. Math. Soc. 6, 459-501 (1993). MR 93k:65045

[10] M. Shub, S. Smale, Complexity of Bézout's Theorem II : Volumes and Probabilities, in : F. Eyssette, A. Galligo Eds. Computational Algebraic Geometry, Progress in Mathematics. Vol. 109, Birkhäuser, 267-285 (1993). MR 94m:68086

[11] M. Shub, S. Smale, Complexity of Bézout's Theorem III : Condition Number and Packing, J. of Complexity, 9, 4-14 (1993). MR 94g:65152

[12] M. Shub, S. Smale, Complexity of Bézout's Theorem IV : Probability of Success, Extensions, SIAM J. Numer. Anal., 33, 128-148 (1996). MR 97k:65310 
[13] M. Shub, S. Smale, Complexity of Bézout's Theorem V : Polynomial Time, Theoretical Computer Science, 133, 141-164 (1994). [MR 96d:65091]

[14] S. Smale, Newton's Method Estimates from Data at One Point, in : The Merging of Disciplines : New Directions in Pure, Applied and Computational Mathematics, R. Ewing, K. Gross, C. Martin Eds., Springer, 185-196 (1986). MR 88e:65076

[15] G. Stewart, J. Sun, Matrix Perturbation Theory, Academic Press, 1990. MR 92a:65017

[16] X. Wang, Some results relevant to Smale's reports, in : Proceedings of the Smalefest, M. V. Hirsch, J. E. Marsden, M. Shub Eds., Springer, 456-465 (1993). CMP 94:03

Laboratoire Approximation et Optimisation, Université Paul Sabatier, 31062 Toulouse Cedex 04, France

E-mail address: dedieu@cict.fr

iBM T.J. Watson Research Center, Yorktown Heights, NY 10598-0218, USA

E-mail address: mshub@us.ibm.com 